\title{
Initial Ideals of Tangent Cones to the Richardson Varieties in the Orthogonal Grassmannian
}

\author{
Shyamashree Upadhyay \\ School of Mathematics, Tata Institute of Fundamental Research, Homi Bhabha Road, Colaba, Mumbai 400005, India \\ Correspondence should be addressed to Shyamashree Upadhyay; shyamashree.upadhyay@gmail.com
}

Received 26 October 2012; Accepted 9 January 2013

Academic Editor: Nantel Bergeron

Copyright ( 2013 Shyamashree Upadhyay. This is an open access article distributed under the Creative Commons Attribution License, which permits unrestricted use, distribution, and reproduction in any medium, provided the original work is properly cited.

A Richardson variety $X_{\alpha}^{\gamma}$ in the Orthogonal Grassmannian is defined to be the intersection of a Schubert variety $X^{\gamma}$ in the Orthogonal Grassmannian and an opposite Schubert variety $X_{\alpha}$ therein. We give an explicit description of the initial ideal (with respect to certain conveniently chosen term order) for the ideal of the tangent cone at any $T$-fixed point of $X_{\alpha}^{\gamma}$, thus generalizing a result of Raghavan and Upadhyay (2009). Our proof is based on a generalization of the Robinson-Schensted-Knuth (RSK) correspondence, which we call the Orthogonal-bounded-RSK (OBRSK).

\section{Introduction}

The Orthogonal Grassmannian is defined in Section 2. A Richardson variety $X_{\alpha}^{\gamma}$ in the Orthogonal Grassmannian (The Richardson varieties in the ordinary Grassmannian are also studied by Stanley in [1], where these varieties are called skew Schubert varieties. Discussion of these varieties in the ordinary Grassmannian also appears in [2].) is defined to be the intersection of a Schubert variety $X^{\gamma}$ in the Orthogonal Grassmannian with an opposite Schubert variety $X_{\alpha}$ therein. In particular, the Schubert and opposite Schubert varieties are special cases of the Richardson varieties. In this paper, we provide an explicit description of the initial ideal (with respect to certain conveniently chosen term order) for the ideal of the tangent cone at any $T$-fixed point $e_{\beta}$ of $X_{\alpha}^{\gamma}$. It should be noted that the local properties of the Schubert varieties at $T$-fixed points determine their local properties at all other points, because of the $B$ action; but this does not extend to the Richardson varieties, since Richardson varieties only have a $T$-action.

In Raghavan and Upadhyay [3], an explicit description of the initial ideal (with respect to certain conveniently chosen term orders) for the ideal of the tangent cone at any $T$-fixed point of a Schubert variety in the Orthogonal Grassmannian has been obtained. In this paper, we generalize the result of [3] to the case of the Richardson varieties in the Orthogonal Grassmannian.

Sturmfels [4] and Herzog and Trung [5] proved results on a class of determinantal varieties which are equivalent to the results of [6-8] for the case of the Schubert varieties (in the ordinary Grassmannian) at the $T$-fixed point $e_{\mathrm{id}}$. The key to their proofs was to use a version of the Robinson-SchenstedKnuth correspondence (which we will call the "ordinary" RSK) in order to establish a degree-preserving bijection between a set of monomials defined by an initial ideal and a "standard monomial basis." The difficulty in extending this method of proof to the case of the Schubert varieties (in the ordinary Grassmannian) at an arbitrary $T$-fixed point $e_{\beta}$ lies in generalizing this bijection, which is done in the three papers [6-8]; the work done in [8] is slightly more general, since it applies to the Richardson varieties, and not just to the Schubert varieties. These three bijections, when restricted to the Schubert varieties in the ordinary Grassmannian, are in fact the same bijection (This supports the conviction of the authors in [6] that this bijection is natural and that it is in some sense the only natural bijection satisfying the required geometric conditions.), although this is not immediately apparent. In the work of Kreiman in [8], this "generalized 
bijection" has been viewed for the first time as an extended version of the "ordinary" RSK correspondence, which he calls the Bounded-RSK correspondence; this viewpoint was not present in $[6,7]$. Although the formulations of the bijections in $[6,7]$ are similar to each other, the formulation of the bijection in [8] is in terms of different combinatorial indexing sets.

The work done in [3] by Raghavan and Upadhyay does not involve any version of the RSK correspondence, unlike the work done by Herzog and Trung in [5]. The work done in [3] relies on a degree-preserving bijection between a set of monomials defined by an initial ideal and a "standard monomial basis," and this bijection is proved by Raghavan and Upadhyay in [9]. It is mentioned in [9] that it will be nice if the bijection proved therein can be viewed as a kind of "Bounded-RSK" correspondence, as done by Kreiman in [8] for the case of the Richardson varieties in the ordinary Grassmannian. This paper fulfills the expectation of [9] that one should be able to view the bijection there as a generalized-bounded-RSK correspondence, which we call here the Orthogonal-bounded-RSK correspondence (OBRSK). Kreiman has mentioned in his paper [8] that he believes in the possibility of adapting the methods of his paper $([8])$ to the Richardson varieties in the Symplectic and the Orthogonal Grassmannian as well; this paper supports Kreiman's belief for the Orthogonal Grassmannian case.

\section{The Orthogonal Grassmannian and Richardson Varieties in It}

Fix an algebraically closed field $\mathfrak{t}$ of characteristic not equal to 2 . Fix a natural number $d$, a vector space $V$ of dimension $2 d$ over $\mathfrak{f}$, and a nondegenerate symmetric bilinear form $\langle$,$\rangle on V$. For $k$ an integer such that $1 \leq k \leq 2 d$, set $k^{*}:=2 d+1-k$. Fix a basis $e_{1}, \ldots, e_{2 d}$ of $V$ such that $\left\langle e_{i}, e_{k}\right\rangle$ equals 1 if $i=k^{*}$ and is 0 otherwise. Denote by $S O(V)$ the group of linear automorphisms of $V$ that preserve the bilinear form $\langle$,$\rangle and also the volume form. A linear$ subspace of $V$ is said to be isotropic, if the bilinear form $\langle$,$\rangle vanishes identically on it. Denote by \mathfrak{M}_{d}(V)^{\prime}$ the closed subvariety of the Grassmannian of $d$-dimensional subspaces consisting of the points corresponding to maximal isotropic subspaces. The action of $S O(V)$ on $V$ induces an action on $\mathfrak{M}_{d}(V)^{\prime}$. There are two orbits for this action. These orbits are isomorphic: acting by a linear automorphism that preserves the form but not the volume form gives an isomorphism. We denote by $\mathfrak{M}_{d}(V)$ the orbit of the span of $e_{1}, \ldots, e_{d}$ and call it the (even) Orthogonal Grassmannian. One can define the Orthogonal Grassmannian in the case when the dimension of $V$ is not necessarily even. But it is enough to consider the case when the dimension of $V$ is even, the reason being the following (see also Section 2.1 of [9] for the same ): suppose that the dimension of $V$ is odd; say dimension of $V=2 d+1$. Let $\widetilde{n}:=2 d+2$ and $\widetilde{V}$ be a vector space of dimension $\widetilde{n}$ with a nondegenerate symmetric form. Let $\widetilde{e}_{1}, \ldots, \widetilde{e}_{\widetilde{n}}$ be a basis of $\widetilde{V}$ such that $\left\langle\widetilde{e}_{i}, \widetilde{e}_{k}\right\rangle$ equals 1 if $i=k^{*}$ and is 0 otherwise. Put $e:=\widetilde{e}_{d+1}$ and $f:=\widetilde{e}_{d+2}$. Take $\lambda$ to be an element of the field such that $\lambda^{2}=1 / 2$. We can take $V$ to be the subspace of $\widetilde{V}$ spanned by the vectors $\widetilde{e}_{1}, \ldots, \widetilde{e}_{d}, \lambda e+\lambda f, \widetilde{e}_{d+3}, \ldots$, and $\widetilde{e}_{\tilde{n}}$, and a basis of $V$ to be these vectors in that order.

There is a natural map from $\mathfrak{M}_{d+1}(\widetilde{V})^{\prime}$ to $\mathfrak{M}_{d}(V)$ : intersecting with $V$ an isotropic subspace of $\widetilde{V}$ of dimension $d+1$ gives an isotropic subspace of $V$ of dimension $d$, and we denote this map by $\cap$. The map $\cap$ is onto, for every isotropic subspace of $\widetilde{V}$ (and hence of $V$ ) is contained in an isotropic subspace of $\widetilde{V}$ of dimension $d+1$. In fact, more is true: the map $\cap$ is two-to-one. The map $\cap$ being two-to-one, it is also elementary to see that the two points in any fiber lie one in each component of $\mathfrak{M}_{d+1}(\widetilde{V})^{\prime}$. We therefore get a natural isomorphism between $\mathfrak{M}_{d+1}(\widetilde{V})$ and $\mathfrak{M}_{d}(V)$. Therefore, now onwards we call the (even) Orthogonal Grassmannian $\mathfrak{M}_{d}(V)$ (as defined above for a $2 d$-dimensional vector space $V)$ the Orthogonal Grassmannian.

Let $\mathfrak{M}_{d}(V) \subseteq G_{d}(V) \hookrightarrow \mathbb{P}\left(\wedge^{d} V\right)$ be the Plücker embedding (where $G_{d}(V)$ denotes the Grassmannian of all $d$-dimensional subspaces of $V)$. Thus $\mathfrak{M}_{d}(V)$ is a closed subvariety of the projective variety $G_{d}(V)$, and hence $\mathfrak{M}_{d}(V)$ inherits the structure of a projective variety.

We take $B$ (resp., $B^{-}$) to be the subgroup of $S O(V)$ consisting of those elements that are upper triangular (resp., lower triangular) with respect to the basis $e_{1}, \ldots, e_{2 d}$ and the subgroup $T$ of $S O(V)$ consisting of those elements that are diagonal with respect to $e_{1}, \ldots, e_{2 d}$. It can be easily checked that $T$ is a maximal torus of $S O(V) ; B$ and $B^{-}$are the Borel subgroups of $S O(V)$ which contain $T$. The group $S O(V)$ acts transitively on $\mathfrak{M}_{d}(V)$, and the $T$-fixed points of $\mathfrak{M}_{d}(V)$ under this action are easily seen to be of the form $\left\langle e_{i_{1}}, \ldots, e_{i_{d}}\right\rangle$ for $\left\{i_{1}, \ldots, i_{d}\right\}$ in $I(d)$, where $I(d)$ is the set of subsets of $\{1, \ldots, 2 d\}$ of cardinality $d$ satisfying the following two conditions:

(i) for each $k, 1 \leq k \leq 2 d$, the subset contains exactly one of $k, k^{*}$, and

(ii) the number of elements in the subset that exceed $d$ is even.

We write $I(d, 2 d)$ for the set of all $d$-element subsets of $\{1, \ldots, 2 d\}$. There is a natural partial order on $I(d, 2 d)$ and so also on $I(d)$ : $v=\left(v_{1}<\cdots<v_{d}\right) \leq w=\left(w_{1}<\cdots<w_{d}\right)$ if and only if $v_{1} \leq w_{1}, \ldots, v_{d} \leq w_{d}$. For $\mu=\left\{\mu_{1}, \ldots, \mu_{d}\right\} \in$ $I(d, 2 d), \mu_{1}<\cdots<\mu_{d}$, define the complement of $\mu$ as $\{1, \ldots, 2 d\} \backslash \mu$, and denote it by $\bar{\mu}$.

The $B$-orbits (as well as $B^{-}$-orbits) of $\mathfrak{M}_{d}(V)$ are naturally indexed by its $T$-fixed points: each $B$-orbit (as well as $B^{-}$orbit) contains one and only one such point. Let $\alpha \in I(d)$ be arbitrary, and let $e_{\alpha}$ denote the corresponding $T$-fixed point of $\mathfrak{M}_{d}(V)$. The Zariski closure of the $B$ - (resp., $B^{-}$-) orbit through $e_{\alpha}$, with canonical reduced scheme structure, is called a Schubert variety (resp., opposite Schubert variety) and denoted by $X^{\alpha}$ (resp., $X_{\alpha}$ ). For $\alpha, \gamma \in I(d)$, the schemetheoretic intersection $X_{\alpha}^{\gamma}=X_{\alpha} \cap X^{\gamma}$ is called a Richardson variety. Each $B$-orbit (as well as $B^{-}$-orbit) being irreducible and open in its closure, it follows that $B$-orbit closures (resp., $B^{-}$-orbit closures) are indexed by the $B$-orbits (resp., $B^{-}$orbits). Thus the set $I(d)$ becomes an indexing set for the Schubert varieties in $\mathfrak{M}_{d}(V)$, and the set consisting of all pairs of elements of $I(d)$ becomes an indexing set for the 


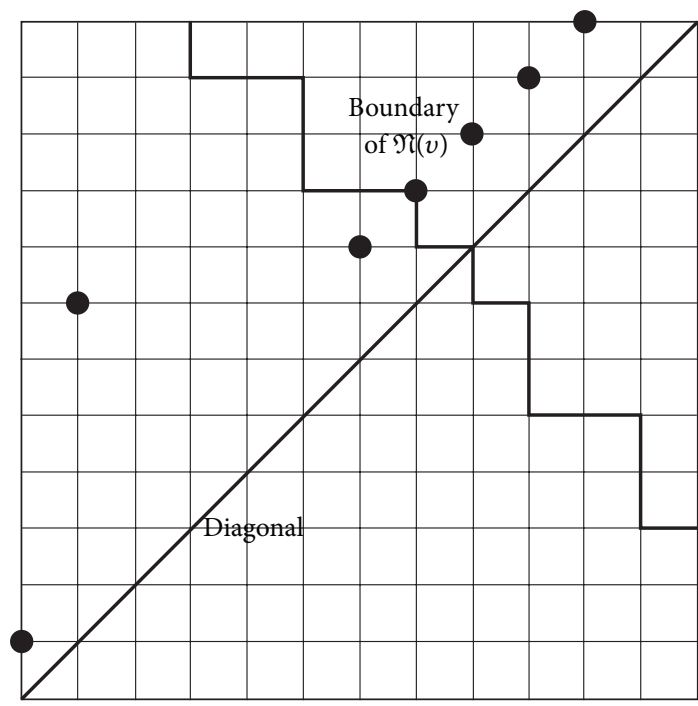

FIGURE 1

Richardson varieties in $\mathfrak{M}_{d}(V)$. It can be shown that $X_{\alpha}^{\gamma}$ is nonempty if and only if $\alpha \leq \gamma$; that for $\beta \in I(d), e_{\beta} \in X_{\alpha}^{\gamma}$ if and only if $\alpha \leq \beta \leq \gamma$.

\section{Statement of the Problem and the Strategy of the Proof}

3.1. Initial Statement of the Problem. The problem that is tackled in this paper is this: given a $T$-fixed point on a Richardson variety in $\mathfrak{M}_{d}(V)$, compute the initial ideal, with respect to some convenient term order, of the ideal of functions vanishing on the tangent cone to the Richardson variety at the given $T$-fixed point. The term order is specified in Section 3.4, and the answer is given in Theorem 7.

For the rest of this paper, $\alpha, \beta$, and $\gamma$ are arbitrarily fixed elements of $I(d)$ such that $\alpha \leq \beta \leq \gamma$. So, the problem tackled in this paper can be restated as follows. Given the Richardson variety $X_{\alpha}^{\gamma}$ in $\mathfrak{M}_{d}(V)$ and the $T$-fixed point $e_{\beta}$ in it, find the initial ideal of the ideal of functions vanishing on the tangent cone at $e_{\beta}$ to $X_{\alpha}^{\gamma}$, with respect to some conveniently chosen term order. The tangent cone being a subvariety of the tangent space at $e_{\beta}$ to $\mathfrak{M}_{d}(V)$, we first choose a convenient set of coordinates for the tangent space. But for that we need to fix some notation.

3.2. Basic Notation. For this subsection, let us fix an arbitrary element $v$ of $I(d, 2 d)$. We will be dealing extensively with ordered pairs $(r, c), 1 \leq r, c \leq 2 d$, such that $r$ is not and $c$ is an entry of $v$. Let $\mathfrak{R}(v)$ denote the set of all such ordered pairs, and set $\mathfrak{N}(v):=\{(r, c) \in \mathfrak{R}(v) \mid r>c\}, \mathfrak{D}(v):=$ $\left\{(r, c) \in \mathfrak{R}(v) \mid r<c^{*}\right\}, \mathfrak{D} \mathfrak{N}(v):=\{(r, c) \in \mathfrak{R}(v) \mid r>$ $\left.c, r<c^{*}\right\}=\mathfrak{D} \mathfrak{R}(v) \cap \mathfrak{N}(v), \mathfrak{d}^{v}:=\left\{(r, c) \in \mathfrak{R}(v) \mid r=c^{*}\right\}$, $\mathfrak{A} \mathfrak{R}(v):=\left\{(r, c) \in \mathfrak{R}(v) \mid r>c^{*}\right\}$, and $\mathfrak{A} \mathfrak{N}(v):=\{(r, c) \in$ $\left.\mathfrak{R}(v) \mid r>c, r>c^{*}\right\}$. We will refer to $\mathfrak{D}^{v}$ as the diagonal.
Figure 1 shows a drawing of $\mathfrak{R}(v)$. We think of $r$ and $c$ in $(r, c)$ as row index and column index, respectively. The columns are indexed from left to right by the entries of $v$ in ascending order and the rows from top to bottom by the entries of $\{1, \ldots, 2 d\} \backslash v$ in ascending order. The points of $\mathfrak{D}^{v}$ are those on the diagonal, the points of $\mathfrak{D} \mathfrak{R}(v)$ are those that are (strictly) above the diagonal, and the points of $\mathfrak{N}(v)$ are those that are to the South-West of the polyline captioned "boundary of $\mathfrak{N}(v)$ "-we draw the boundary so that points on the boundary belong to $\mathfrak{N}(v)$. The reader can readily verify that $d=13$ and $v=$ $(1,2,3,4,6,7,10,11,13,15,18,19$, and 22$)$ for the particular picture drawn. The points of $\mathfrak{D} \mathfrak{R}(v)$ indicated by solid circles form an extended $v$-chain (see Figure 1); the definition of an extended $v$-chain is given later in Section 3.5.

We will be considering monomials in some of these sets. A monomial, as usual, is a subset with each member being allowed a multiplicity (taking values in the nonnegative integers). The degree of a monomial has also the usual sense: consider the underlying set of the monomial and look at the multiplicity with which each element of this underlying set appears in the monomial; the degree of the monomial is the sum of these multiplicities. The intersection of any two monomials in a set also has the natural meaning; it is the monomial whose underlying set is the intersection of the underlying sets of the given two monomials and the multiplicity of each element being the minimum of the multiplicities with which the element occurs in the two given monomials.

Given any two monomials $A$ and $B$ consisting of elements of $\mathfrak{R}(v)$, let set $(A)$ and set $(B)$ denote the underlying sets of $A$ and $B$, respectively. We say that $B \subseteq A$ (as monomials) if set $(B) \subseteq \operatorname{set}(A)$, and the multiplicity with which every element occurs in the monomial $B$ is less than or equal to the multiplicity with which the same element occurs in the monomial $A$. Given two monomials $A$ and $B$ consisting of elements of $\mathfrak{R}(v)$ such that $B \subseteq A$ (as monomials), we can define a monomial called the "monomial minus" of $B$ from $A$ (denoted by $A \backslash_{m} B$ ) as follows. Take any element $x$ of $\operatorname{set}(B)$. If the multiplicity with which $x$ occurs in $A$ is $m_{x}(A)$ and the multiplicity with which $x$ occurs in $B$ is $m_{x}(B)$, then the multiplicity with which $x$ occurs in the monomial $A \backslash_{m} B$ is $m_{x}(A)-m_{x}(B)$. And any element in $\operatorname{set}(A) \backslash \operatorname{set}(B)$ occurs in the monomial $A \backslash_{m} B$ with the same multiplicity with which it occurs in $A$. This finishes the description of $A \backslash_{m} B$.

Remark 1 . Note that in this subsection, and $v$ was any element of $I(d, 2 d), v$ was not necessarily in $I(d)$. In particular, all the above basic notations will hold true, if we take $v \in I(d)$ as well.

3.3. The Ideal of the Tangent Cone to $X_{\alpha}^{\gamma}$ at $e_{\beta}$. Let $\beta$ be the element of $I(d)$ which was fixed at the beginning of this section. Consider the matrix of size $2 d \times d$ whose columns are numbered by the entries of $\beta$ and the rows by $\{1, \ldots, 2 d\}$; the rows corresponding to the entries of $\beta$ form the $d \times d$ identity matrix, and the remaining $d$ rows form a skew symmetric 
matrix whose upper half entries are variables of the form $X_{(r, c)}$, where $(r, c) \in \mathfrak{D} \mathfrak{R}(\beta)$.

Let $\mathfrak{M}_{d}(V) \subseteq G_{d}(V) \hookrightarrow \mathbb{P}\left(\wedge^{d} V\right)$ be the Plücker embedding (where $G_{d}(V)$ denotes the Grassmannian of all $d$ dimensional subspaces of $V)$. For $\theta$ in $I(d, 2 d)$, let $p_{\theta}$ denote the corresponding Plücker coordinate. Consider the affine patch $\mathbb{A}$ of $\mathbb{P}\left(\wedge^{d} V\right)$ given by $p_{\beta} \neq 0$. The affine patch $\mathbb{A}^{\beta}:=$ $\mathfrak{M}_{d}(V) \cap \mathbb{A}$ of the Orthogonal Grassmannian $\mathfrak{M}_{d}(V)$ is an affine space whose coordinate ring can be taken to be the polynomial ring in variables of the form $X_{(r, c)}$ with $(r, c) \in$ $\mathfrak{D}(\beta)$.

For $\theta \in I(d)$, consider the submatrix of the above mentioned matrix given by the rows numbered $\theta \backslash \beta$ and columns numbered $\beta \backslash \theta$. Such a submatrix is of even size and is skewsymmetric about antidiagonal, therefore its determinant is a square. The square root, which is determined up to sign, is called the Pfaffian. Let $f_{\theta, \beta}$ denote this Pfaffian. Set $Y_{\alpha}^{\gamma}(\beta):=$ $X_{\alpha}^{\gamma} \cap \mathbb{A}^{\beta}$. From [10] we can deduce a set of generators for the ideal $I$ of functions on $\mathbb{A}^{\beta}$ vanishing on $Y_{\alpha}^{\gamma}(\beta)$ (see Section 4.2 of [9] for the special case of the Schubert varieties). The following equation gives the generators:

$$
I=\left(f_{\tau, \beta} \mid \tau \in I(d), \alpha \nless \tau \text { or } \tau \nless \xi \gamma\right) .
$$

We are interested in the tangent cone to $X_{\alpha}^{\gamma}$ at $e_{\beta}$ or, what is the same, the tangent cone to $Y_{\alpha}^{\gamma}(\beta)$ at the origin. Observe that $f_{\tau, \beta}$ is a homogeneous polynomial. Because of this, $Y_{\alpha}^{\gamma}(\beta)$ itself is a cone and so is equal to its tangent cone at the origin. The ideal of the tangent cone to $X_{\alpha}^{\gamma}$ at $e_{\beta}$ is therefore the ideal $I$ in (1).

3.4. The Term Order. We now specify the term order $\triangleright$ on monomials in the coordinate functions (of the tangent space to $\mathfrak{M}_{d}(V)$ at the torus fixed point $e_{\beta}$; it is easy to see that coordinate ring of the tangent space to $\mathfrak{M}_{d}(V)$ at $e_{\beta}$ is the polynomial ring in variables of the form $X_{(r, c)}$, where $(r, c) \in$ $\mathfrak{Q}(\beta)$ ) with respect to which the initial ideal of the ideal $I$ of the tangent cone is to be taken.

Let > be a total order on $\mathfrak{D R}(\beta)$ satisfying all of the following 6 conditions.

(i) $\mu>v$, if $\mu \in \mathfrak{D} \mathfrak{N}(\beta), v \in \mathfrak{D} \mathfrak{R}(\beta) \backslash \mathfrak{D} \mathfrak{N}(\beta)$, and the row indices of $\mu$ and $v$ are equal.

(ii) $\mu>v$, if $\mu \in \mathfrak{D N}(\beta), v \in \mathfrak{D} \mathfrak{N}(\beta)$, the row indices of $\mu$ and $\nu$ are equal, and the column index of $\mu$ exceeds that of $v$.

(iii) $\mu>v$ if $\mu \in \mathfrak{D} \mathfrak{N}(\beta), v \in \mathfrak{D} \mathfrak{R}(\beta)$, and the row index of $\mu$ is less than that of $\nu$.

(iv) $\mu>v$, if $\mu \in \mathfrak{D} \mathfrak{R}(\beta) \backslash \mathfrak{D} \mathfrak{N}(\beta), v \in \mathfrak{D} \mathfrak{N}(\beta)$, and the column indices of $\mu$ and $v$ are equal.

(v) $\mu>v$, if $\mu \in \mathfrak{D} \mathfrak{R}(\beta) \backslash \mathfrak{D} \mathfrak{N}(\beta), v \in \mathfrak{D} \mathfrak{R}(\beta) \backslash \mathfrak{D} \mathfrak{N}(\beta)$, the column indices of $\mu$ and $v$ are equal, and the row index of $\mu$ exceeds that of $\nu$.

(vi) $\mu>v$, if $\mu \in \mathfrak{D} \mathfrak{R}(\beta) \backslash \mathfrak{D} \mathfrak{N}(\beta), v \in \mathfrak{D} \mathfrak{R}(\beta)$, and the column index of $\mu$ is less than that of $\nu$.
Note here that the first 3 conditions above are the same as the conditions put on the total order $>_{1}$ as mentioned in Section 1.6 of [3]. Recall that in the paper [3], initial ideals of ideals of tangent cones at torus fixed points to the Schubert varieties in Orthogonal Grassmannians were computed, and the paper [3] did not deal with the Richardson varieties. The last 3 conditions above arise in this paper as an addition to the 3 conditions put on the total order $>_{1}$ (as mentioned in Section 1.6 of [3]), because here we are dealing with the Richardson varieties in $\mathfrak{M}_{d}(V)$.

Let $\triangleright$ be the term order on monomials in $\mathfrak{D} \mathfrak{R}(\beta)$ given by the homogeneous lexicographic order with respect to $>$.

Remark 2. The total order $>$ on $\mathfrak{D} \Re(\beta)$ satisfying the 6 properties mentioned above can be realized as a concrete total order on $\mathfrak{D}(\beta)$ if we put the following extra condition on it.

Let $r(\mu), r(v), c(\mu)$, and $c(v)$ denote the row index of $\mu$, the row index of $\nu$, the column index of $\mu$, and the column index of $\nu$, respectively. If $r(\mu)<r(\nu), \mu \in \mathfrak{D} \mathfrak{R}(\beta) \backslash \mathfrak{D} \mathfrak{N}(\beta)$, $v \in \mathfrak{D} \mathfrak{N}(\beta)$, and $c(v)<c(\mu)$, then $\nu>\mu$ when $(r(v), c(\mu)) \notin$ $\mathfrak{N}(\beta)$ and $\mu>v$ when $(r(\nu), c(\mu)) \in \mathfrak{N}(\beta)$.

3.5. Extended $v$-Chains and Associated Elements of $I(d)$. For this subsection, let $v$ be an arbitrarily fixed element of $I(d, 2 d)$ (not necessarily an element of $I(d)$, unless otherwise stated). For elements $\lambda=(R, C), \mu=(r, c)$ of $\mathfrak{R}(v)$, we write $\lambda>\mu$ if $R>r$ and $C<c$ (Note that these are strict inequalities.). A sequence $\lambda_{1}>\cdots>\lambda_{k}$ of elements of $\mathfrak{D} \mathfrak{R}(v)$ is called an extended $v$-chain. Note that an extended $v$-chain can also be empty. Letting $C$ to be an extended $v$-chain, we define $C^{+}:=$ $C \cap \mathfrak{D}(v)$ and $C^{-}:=C \cap(\mathfrak{R}(v) \backslash \mathfrak{D} \mathfrak{N}(v))$. We call $C^{+}$(resp., $C^{-}$) the positive (resp., negative) parts of the extended $v$-chain $C$. We call an extended $v$-chain $C$ positive (resp., negative) if $C=C^{+}$(resp., $C=C^{-}$). The extended $v$-chain $C$ is called nonempty if it has at least one element in it. Note that if we specialize to the case when $v \in I(d)$, then whatever is called a $v$-chain in Section 2.4.1 of [9] is a positive extended $v$-chain over here. To every extended $v$-chain $C$, we will now associate 2 subsets $\mathfrak{d}_{C}^{v}(+)$ and $\mathfrak{d}_{C}^{v}(-)$ of $\mathfrak{d}^{v}$ (each of even cardinality), but for that we first need to fix some notation and recall certain terminology from $[3,9]$.

Definition 3 (Pr and Pro). Given any subset $D$ of $\mathfrak{D} \mathfrak{N}(v)$, let $\operatorname{Pr}(D)$ denote the monomial whose underlying set is given by $\{\mu \mid \mu$ is a vertical or horizontal projection of some element of $D\}$ (both vertical and horizontal, as defined in Section 2.3 of [9]), and the cardinality of any element $\mu$ of this underlying set inside the monomial $\operatorname{Pr}(D)$ equals the number of elements in $D$ whose one of the projections (vertical or horizontal) is $\mu$. For $\lambda=(r, c)$ in $\mathfrak{R}(v)$, define $\lambda^{\#}:=\left(c^{*}, r^{*}\right)$. The involution $\lambda \mapsto \lambda^{\#}$ is just the reflection with respect to the diagonal $\mathfrak{d}^{v}$. For a subset $\mathfrak{E}$ of $\mathfrak{N}(v)$, the symbol $\mathfrak{E}^{\#}$ has the obvious meaning. One calls $\mathfrak{E}$ symmetric if $\mathfrak{E}=\mathfrak{F}^{\#}$. Given any symmetric subset $E$ of $\mathfrak{N}(v)$, let one denote by $E$ (top) the set $E \cap \mathfrak{D} \mathfrak{N}(v)$, by $E(\operatorname{diag})$ the set $E \cap \mathfrak{d}^{v}$, and by $\operatorname{Pro}(E)$ the monomial formed by taking the union of the subset $E$ (diag) with the monomial $\operatorname{Pr}(E$ (top)). Let one make the definition of 
$\operatorname{Pro}(E)$ more precise. The multiplicity with which any element occurs in the monomial $\operatorname{Pro}(E)$ is equal to the sum of the multiplicities with which the element occurs in the subset $E$ (diag) and the monomial $\operatorname{Pr}(E$ (top)). So for any symmetric subset $E$ of $\mathfrak{N}(v)$, $\operatorname{Pro}(E)$ is a monomial consisting of elements from the diagonal. Similarly for any subset $D$ of $\mathfrak{D} \mathfrak{N}(v), \operatorname{Pr}(D)$ is also a monomial consisting of elements from the diagonal.

Let $v \in I(d)$. Let $C: \alpha_{1}=\left(r_{1}, c_{1}\right)>\alpha_{2}=\left(r_{2}, c_{2}\right)>\cdots>$ $\alpha_{l}=\left(r_{l}, c_{l}\right)$ be a positive extended $v$-chain in $\mathfrak{D} \mathfrak{N}(v)$. Two consecutive elements $\alpha_{j}$ and $\alpha_{j+1}$ of $C$ are said to be connected if the following conditions are both satisfied:

(i) their legs are "intertwined", that is, $r_{j}^{*} \geq c_{j+1}$,

(ii) the point $\left(r_{j+1}, r_{j}^{*}\right)$ belongs to $\mathfrak{N}(v)$.

Consider the coarsest equivalence relation on the elements of $C$ generated by the above relation. The equivalence classes of $C$ with respect to this equivalence relation are called the connected components of the $v$-chain $C$.

For any $\alpha=(r, c)$ in $\mathfrak{D} \mathfrak{N}(v)$, the elements $p_{v}(\alpha):=\left(c^{*}, c\right)$ and $p_{h}(\alpha):=\left(r, r^{*}\right)$ of the diagonal are called, respectively, the vertical and horizontal projections of $\alpha$. Given the positive extended $v$-chain $C$ as above, let $\widetilde{\Xi}_{C}$ be the monomial of $\mathfrak{N}(v)$ associated to $C$ defined as follows.

First suppose that $C$ is a connected $v$-chain in $\mathfrak{D} \mathfrak{N}(v)$. Observe that if there is at all an integer $j(1 \leq j \leq l)$ such that the horizontal projection $p_{h}\left(\alpha_{j}\right)$ does not belong to $\mathfrak{N}(v)$, then $j=l$. Define

$$
\mathfrak{S}_{C}:=\left\{\begin{array}{cc}
\left\{p_{v}\left(\alpha_{1}\right), \ldots, p_{v}\left(\alpha_{l}\right)\right\} & \text { if } l \text { is even } \\
\left\{p_{v}\left(\alpha_{1}\right), \ldots, p_{v}\left(\alpha_{l}\right)\right\} \cup\left\{p_{h}\left(\alpha_{l}\right)\right\} & \\
& \text { if } l \text { is odd, } p_{h}\left(\alpha_{l}\right) \in \mathfrak{N}(v) \\
\left\{p_{v}\left(\alpha_{1}\right), \ldots, p_{v}\left(\alpha_{l-1}\right)\right\} \cup\left\{\alpha_{l}, \alpha_{l}^{\#}\right\} & \\
& \text { if } l \text { is odd, } p_{h}\left(\alpha_{l}\right) \notin \mathfrak{N}(v) .
\end{array}\right.
$$

For a $v$-chain $C$ that is not necessarily connected, let $C=C_{1} \cup C_{2} \cup \cdots$ be the partition of $C$ into its connected components, and set

$$
\mathfrak{S}_{C}:=\mathfrak{S}_{C_{1}} \cup \mathfrak{S}_{C_{2}} \cup \cdots
$$

Note that even if we take $v$ to be in $I(d, 2 d)$ (and not merely in $I(d))$ and define $\mathfrak{S}_{C}$ for any positive extended $v$ chain $C$ exactly in the same way as we did above, there is no logical inconsistency. Hence we extend the definition of $\widetilde{S}_{C}$ to any positive extended $v$-chain $C$, where $v \in I(d, 2 d)$. Clearly $\mathfrak{S}_{C}$ is a symmetric subset of $\mathfrak{N}(v)$. Hence the monomial $\operatorname{Pro}\left(\mathfrak{S}_{C}\right)$ is well defined for any positive extended $v$-chain $C$, where $v \in I(d, 2 d)$.

Definition 4 (the flip map $F$ ). For any $v \in I(d, 2 d)$ and any element $\lambda=(r, c) \in \mathfrak{R}(v)$, let $F(\lambda)$ be the element of $\mathfrak{R}\left(v^{*}\right)$ given by $F(\lambda):=(c, r)$. So $F$ is an invertible map from $\mathfrak{R}(v)$ to $\mathfrak{R}\left(v^{*}\right)$ (note here that if $v \in I(d)$, then $v^{*}$ needs not always to belong to $I(d))$; let one denote the inverse of $F$ by $F^{-1}$. The map $F$ naturally induces an invertible map from the set of all monomials in $\mathfrak{R}(v)$ to the set of all monomials in $\mathfrak{R}\left(v^{*}\right)$. One continues to call the induced map $F$ and its inverse $F^{-1}$.
Definition 5 (the subsets $\mathfrak{d}_{C}^{v}(+)$ and $\mathfrak{d}_{C}^{v}(-)$ of $\left.\mathfrak{d}^{v}\right)$. Given any non-empty extended $v$-chain $C$, one will now associate 2 subsets $\mathfrak{d}_{C}^{v}(+)$ and $\mathfrak{d}_{C}^{v}(-)$ of $\mathfrak{d}^{v}$ (each of even cardinality) with it. Let

$$
\mathfrak{D}_{C}^{v}(+):=\left\{\begin{aligned}
& F^{-1}\left(\operatorname{Pr}(F(C)) \backslash_{m} \operatorname{Pro}\left(\mathfrak{S}_{F(C)}\right)\right) \\
& \text { if } C \text { is negative } \\
& \operatorname{Pro}\left(\mathfrak{S}_{C^{+}}\right) \text {if otherwise. }
\end{aligned}\right.
$$

Similarly, let

$$
\mathfrak{d}_{C}^{v}(-):= \begin{cases}\operatorname{Pr}(C) \backslash_{m} \operatorname{Pro}\left(\mathfrak{\Xi}_{C}\right) & \text { if } C \text { is positive } \\ F^{-1}\left(\operatorname{Pro}\left(\mathfrak{S}_{F\left(C^{-}\right)}\right)\right) & \text {if otherwise. }\end{cases}
$$

It is an easy exercise to check that $\mathfrak{d}_{C}^{v}(+)$ and $\mathfrak{d}_{C}^{v}(-)$ thus defined are actually subsets (not monomials) of $\boldsymbol{b}^{v}$ and that each of them has even cardinality.

Definition 6 (elements of $I(d)$ associated with $\mathfrak{d}_{C}^{v}(+)$ and $\left.\mathfrak{D}_{C}^{v}(-)\right)$. For this definition, we let $v$ to be an arbitrary element in $I(d)$. Note that given any subset $S$ of $\mathfrak{d}^{v}$ of even cardinality, one can naturally associate an element of $I(d)$ to it by removing those entries from $v$ which appear as column indices in the elements of the set $S$ and then adding to it the row indices of all the elements of $S$. It is easy to check that the resulting element actually belongs to $I(d)$. One denotes the resulting element by $I(d)(S)$. If $S$ is empty, then $I(d)(S)$ is taken to be $v$ itself.

Let $w_{C}^{+}(v):=I(d)\left(\mathfrak{D}_{C}^{v}(+)\right)$ and $w_{C}^{-}(v):=I(d)\left(\mathfrak{D}_{C}^{v}(-)\right)$. These are the two elements of $I(d)$ that we can naturally associate with the subsets $\mathfrak{D}_{C}^{v}(+)$ and $\mathfrak{D}_{C}^{v}(-)$ of $\mathfrak{d}^{v}$.

3.6. The Main Theorem and a Strategy of the Proof. Recall that the ideal of the tangent cone to $X_{\alpha}^{\gamma}$ at $e_{\beta}$ is the ideal $I$ given by (1). Let $\triangleright$ be as in Section 3.4. For any element $f \in I$; let in $f$ denote the initial term of $f$ with respect to the term order $\triangleright$. We define in $\operatorname{in}_{\triangleright} I$ to be the ideal $\left\langle\operatorname{in}_{\triangleright} f \mid f \in I\right\rangle$ inside the polynomial ring $P:=\mathfrak{f}\left[X_{(r, c)} \quad \mid(r, c) \in \mathfrak{D} \mathfrak{R}(\beta)\right]$. For any monomial $U$ in $\mathfrak{D} \Re(\beta)$, let us denote by $X_{U}$ the product of all the elements $X_{(r, c)}$, where $(r, c)$ runs over the elements in $U$ with multiplicities.

Let Chains $_{\alpha}^{\gamma}(\beta)$ denote the set $\left\{X_{C} \mid C\right.$ is a nonempty extended $\beta$-chain in $\mathfrak{D} \Re(\beta)$ such that either (i) or (ii) below holds\}.

(i) $C^{-}$is nonempty, and $\alpha \not w_{C^{-}}^{-}(\beta)$. (ii) $C^{+}$is nonempty, and $w_{C^{+}}^{+}(\beta) \not \gamma$.

The main result of this paper is the following.

Theorem 7. in $_{\triangleright} I=\left\langle\right.$ Chains $\left._{\alpha}^{\gamma}(\beta)\right\rangle$.

Example 8. Let $d=5, \alpha=(1,2,3,6,7), \beta=(1,2,4,6,8)$, and $\gamma=(1,2,5,7,8)$. Clearly then $\alpha \leq \beta \leq \gamma$. It can be verified that the set Chains ${ }_{\alpha}^{\gamma}(\beta)$ consists of elements of the following 3 types.

(i) Any extended $\beta$-chain in $\mathfrak{D} \Re(\beta)$ which contains the element $(3,6)$.

(ii) Any extended $\beta$-chain in $\mathfrak{D} \Re(\beta)$ which contains the element $(3,4)$ and a nonempty positive part. 
(iii) All positive extended $\beta$-chain in $\mathfrak{D} \mathfrak{R}(\beta)$ except the singleton- $\beta$-chain $\{(5,4)\}$.

One can therefore determine the corresponding initial ideal in ${ }_{\triangleright} I$ using Theorem 7.

Remark 9. It follows from the statement of Theorem 7 that the set of all monomials in $\mathfrak{D} \mathfrak{R}(\beta)$ which contain at least one extended $\beta$-chain $C$ such that $X_{C} \in$ Chains $_{\alpha}^{\gamma}(\beta)$ form a vector space basis of the initial ideal in $_{\triangleright} I$ over the field $\mathfrak{f}$; see, for example, Example 8. In the special case when the Richardson variety is a Schubert variety, it is easy to see that the previous statement of this remark says exactly what has been said in the main theorem (Theorem 1.8.1) of [3].

Remark 10. Since in ${ }_{\triangleright} I=\left\langle\right.$ Chains $\left._{\alpha}^{\gamma}(\beta)\right\rangle$, it follows that the dimension as a vector space of the graded piece of $P /$ in $_{\triangleright} I$ of degree $m$ equals the cardinality of the set of all monomials in $\mathfrak{D} \Re(\beta)$ of degree $m$ which do not contain any extended $\beta$-chain $C$ such that $X_{C} \in$ Chains $_{\alpha}^{\gamma}(\beta)$.

Since $P / I$ and $P /$ in $_{\triangleright} I$ have the same Hilbert function, the same is true with $P /$ in $_{\triangleright} I$ (in the previous statement) replaced by $P / I$. Hence if $R_{\alpha}^{\gamma}(\beta)$ denotes the coordinate ring of the tangent cone to $X_{\alpha}^{\gamma}$ at $e_{\beta}$, then the dimension as a vector space of the homogeneous piece of $R_{\alpha}^{\gamma}(\beta)$ of degree $m$ equals the cardinality of the set of all monomials in $\mathfrak{D} \Re(\beta)$ of degree $m$ which are such that for every extended $\beta$-chain $C$ in the monomial, $\alpha \leq w_{C^{-}}^{-}(\beta)$ and $w_{C^{+}}^{+}(\beta) \leq \gamma$.

From Remark 10, the following corollary is immediate (the proof is similar to that of Corollary 2.5.2 of [9])

Corollary 11. The multiplicity of $X_{\alpha}^{\gamma}$ at $e_{\beta}$ equals the number of monomials in $\mathfrak{D} \Re(\beta)$ of maximal cardinality that are squarefree and are such that, given any extended $\beta$-chain $C$ in any of these monomials, one has: $\alpha \leq w_{C^{-}}^{-}(\beta)$ and $w_{C^{+}}^{+}(\beta) \leq \gamma$.

We now briefly sketch the proof of Theorem 7 (omitting details, which can be found in Section 8). In order to introduce the main combinatorial objects of interest and outline a strategy of the proof, we will first need to prove that the set Chains $_{\alpha}^{\gamma}(\beta) \subseteq$ in $_{\triangleright} I$, and this proof will follow from whatever is said in Remark 12.

Remark 12. Let $C$ be a nonempty extended $\beta$-chain in $\mathfrak{R}(\beta)$ such that $X_{C} \in$ Chains $_{\alpha}^{\gamma}(\beta)$. If $C^{+}$is nonempty and $w_{C^{+}}^{+}(\beta) \not$ $\gamma$, then it can be proved that $X_{C^{+}} \in$ in $_{\triangleright} I$, the proof being exactly the same as that in Section 4 of [3]. Then since $i_{\triangleright} I$ is an ideal and $X_{C}=X_{C^{-}} X_{C^{+}}$, it follows that $X_{C} \in$ in $_{\triangleright} I$.

If $C^{-}$is nonempty and $\alpha \not w_{C^{-}}^{-}(\beta)$, look at $F\left(C^{-}\right)$, where $F$ is the flip map as defined in Definition 4 from the set of all monomials in $\mathfrak{R}(\beta)$ to the set of all monomials in $\mathfrak{R}\left(\beta^{*}\right)$. Then $F\left(C^{-}\right)$is a positive extended $\beta^{*}$-chain in $\mathfrak{D} \Re\left(\beta^{*}\right)$. We need to prove that $X_{C} \in \operatorname{in}_{\triangleright} I$, for which it is enough to show that $X_{C^{-}} \in$ in $_{\triangleright} I$. To prove that $X_{C^{-}} \in$ in $_{\triangleright} I$, we will proceed in a way equivalent to the proof done in Section 4 of [3]. But there is a subtle difference between what is proved in Section 4 of [3] and what we are going to prove here; namely, Whatever was proved in Section 4 of [3] can be rephrased in the language of this paper as "Every positive extended $\beta$-chain
$D$ satisfying the property that $w_{D}^{+}(\beta) \nless \gamma$ belongs to the initial ideal of the ideal of the tangent cone," but here we are going to prove that "Every negative extended $\beta$-chain $D$ satisfying the property that $\alpha \nless w_{D}^{-}(\beta)$ belongs to the initial ideal of the ideal of the tangent cone."

Because of this subtle difference, we need to construct certain gadgets for negative extended $\beta$-chains, which will play a role similar to the role of the objects like the new forms, Proj and Proje corresponding to positive extended $\beta$-chains (For positive extended $\beta$-chains, such objects are already defined in [3]). This construction is given in the following paragraph.

Consider the positive extended $\beta^{*}$-chain $F\left(C^{-}\right)$. We can construct new forms, Proj and Proj ${ }^{e}$ for $F\left(C^{-}\right)$in the same way as they were constructed in [3], note here the fact that $\beta^{*}$ may or may not belong to $I(d)$ does not really affect the construction of the new forms, Proj and $\operatorname{Proj}^{e}$ for $F\left(C^{-}\right)$. Then we apply the map $F^{-1}$ to these objects constructed for $F\left(C^{-}\right)$, the resulting objects are the analogues of the new forms, Proj and $\operatorname{Proj}^{e}$ for the negative extended $\beta$-chain $C^{-}$. We apply a similar treatment to any other monomial related to $F\left(C^{-}\right)$ that we happen to encounter if we replace the " $\nu$-chain $A$ " in Section 4.2 of [3] with "the positive extended $\beta^{*}$-chain $F\left(C^{-}\right)$."

In Section 2.4 of [3], an element $y_{E}$ of $I(d)$ corresponding to any $v$-chain $E$ (the notion of a $v$-chain being as in Section 1.7 of [3]) has been defined. The analogous element of $I(d)$ for the negative extended $\beta$-chain $C^{-}$(we call it $y_{C^{-}}$here) can be obtained from $F^{-1}$ ( $\operatorname{Proj}^{e}\left(F\left(C^{-}\right)\right)$) by following the natural process: the column indices of elements of $F^{-1}\left(\operatorname{Proj}^{e}\left(F\left(C^{-}\right)\right)\right)$ occur as members of $\beta$; these are replaced by the row indices to obtain the desired element of $I(d)$ for $C^{-}$. It is easy to check that $y_{C^{-}}$belongs to $I(d)$ and that $y_{C^{-}} \leq w_{C^{-}}^{-}(\beta) \leq \beta$. Since we already have that $\alpha \nless w_{C^{-}}^{-}(\beta)$, it follows that $\alpha \nless y_{C^{-}}$. These facts about $y_{C^{-}}$will be needed to produce an analogue of the main proof of [3] in our present case. To be more precise, these facts about $y_{C^{-}}$give the analogues of Propositions 2.4.1 and 2.4.2 of [3], and these two propositions had been used quite crucially inside the main proof of [3].

With all these analogues constructed for negative extended $\beta$-chains, we can proceed in an "equivalent" manner (Here, by "equivalent" we mean keeping track of the subtle difference as mentioned above and working accordingly.) as in the paper [3] and end up proving the desired fact, namely, $X_{C^{-}} \in \operatorname{in}_{\triangleright} I$.

Since Chains ${ }_{\alpha}^{\gamma}(\beta) \subseteq \operatorname{in}_{\triangleright} I$, we have $\left\langle\right.$ Chains $\left._{\alpha}^{\gamma}(\beta)\right\rangle \subseteq$ in $_{\triangleright} I$. To prove Theorem 7 , we now need to show that $\left\langle\right.$ Chains $\left._{\alpha}^{\gamma}(\beta)\right\rangle \supseteq \operatorname{in}_{\triangleright} I$. For this, it suffices to show that, in any degree, the number of monomials of $\left\langle\right.$ Chains $\left._{\alpha}^{\gamma}(\beta)\right\rangle$ is $\geq$ the number of monomials of in ${ }_{\triangleright} I$. Or equivalently, it suffices to show that, in any degree, the number of monomials of $P /\left\langle\right.$ Chains $\left._{\alpha}^{\gamma}(\beta)\right\rangle$ is $\leq$ the number of monomials of $P /$ in $_{\triangleright} I$.

Consider the affine patch $Y_{\alpha}^{\gamma}(\beta)\left(:=X_{\alpha}^{\gamma} \cap \mathbb{A}^{\beta}\right)$ of the Richardson variety $X_{\alpha}^{\gamma}$. The following is a well known result.

Theorem 13. $\mathfrak{E}\left[Y_{\alpha}^{\gamma}(\beta)\right]=P / I$, where $P=\mathfrak{E}\left[X_{(r, c)} \mid(r, c) \in\right.$ $\mathfrak{D}(\beta)]$ and $I$ is as in (1). 
TABLE 1: Two subsets of the ring $P=\mathfrak{f}\left[X_{(r, c)} \mid(r, c) \in \mathfrak{D} \mathfrak{R}(\beta)\right]$ and their indexing sets.

\begin{tabular}{ll}
\hline Set of elements in $P=\mathfrak{f}\left[X_{(r, c)} \mid(r, c) \in \mathfrak{D} \mathfrak{R}(\beta)\right]$ & Indexing set \\
\hline Monomials of $P /\left\langle\operatorname{Chains}_{\alpha}^{\gamma}(\beta)\right\rangle$ & Pairs of nonempty skew-symmetric lexicographic multisets on $\bar{\beta} \times \beta$ bounded by $T_{\alpha}, W_{\gamma}$ \\
Standard monomials on $Y_{\alpha}^{\gamma}(\beta)$ & Nonvanishing skew-symmetric notched bitableaux on $\bar{\beta} \times \beta$ bounded by $T_{\alpha}, W_{\gamma}$ \\
\hline
\end{tabular}

Both the monomials of $P /$ in $_{\triangleright} I$ and the standard monomials on $Y_{\alpha}^{\gamma}(\beta)$ form a basis for $P / I$, and thus agree in cardinality in any degree. Therefore, to prove, that in any degree, the number of monomials of $P /\left\langle\right.$ Chains $\left.\alpha_{\alpha}^{\gamma}(\beta)\right\rangle$ is $\leq$ the number of monomials of $P /$ in $_{\triangleright} I$, it suffices to give a degree-preserving injection from the set of all monomials in $P /\left\langle\right.$ Chains $\left._{\alpha}^{\gamma}(\beta)\right\rangle$ to the set of all standard monomials on $Y_{\alpha}^{\gamma}(\beta)$. We construct such an injection, the Orthogonal-bounded-RSK (OBRSK), from an indexing set of the former to an indexing set of the later. These indexing sets are given in Table 1.

\section{Skew-Symmetric Notched Bitableaux}

In this section onwards, the terminology and notation of Sections 4 and 5 of [8] will be in force. Recall the definition of a semistandard notched bitableau from Section 5 of [8].

Definition 14 (dual of an element with respect to a semistandard notched bitableau). Let $(P, Q)$ be a semistandard notched bitableau. Let $p_{i, j}$ (resp., $q_{i, j}$ ) denote the entry in the $i$ th row and $j$ th column of $P$ (resp., $Q$ ). For any row number $i$ of $P$ (or of $Q$ ), let $k_{i}$ denote the total number of entries in the $i$ th row of $P$ (or $Q$ ). One calls the entry $q_{i, k_{i}+1-j}$ of $Q$ the dual of the entry $p_{i, j}$ of $P$ with respect to $(P, Q)$. Similarly, we call the entry $p_{i, k_{i}+1-j}$ of $P$ the dual of the entry $q_{i, j}$ of $Q$ with respect to $(P, Q)$.

Note that any entry of $P$ or $Q$ can be identified uniquely by specifying 4 coordinates, namely, the entry $x$, the tableau $A(A=P$ or $Q)$ in which the entry lies, the row number $i$ of the entry in the tableau $A$, and the column number $j$ of the entry in the tableau $A$. Let $\operatorname{set}(P, Q)$ denote the set of all 4tuples of the form $(x, A, i, j)$. Given any 4 -tuple $(x, A, i, j) \in$ $\operatorname{set}(P, Q)$, let us denote by $D_{(P, Q)}(x, A, i, j)$ the 4-tuple which represents the dual of $x$ with respect to $(P, Q)$ (as defined in Definition 14). For $(x, A, i, j),\left(x^{\prime}, A^{\prime}, i^{\prime}, j^{\prime}\right) \in \operatorname{set}(P, Q)$, we say that $(x, A, i, j) \preceq\left(x^{\prime}, A^{\prime}, i^{\prime}, j^{\prime}\right)$ if $x \leq x^{\prime}$. Similarly, we say that $(x, A, i, j) \prec\left(x^{\prime}, A^{\prime}, i^{\prime}, j^{\prime}\right)$ if $x<x^{\prime}$ and $(x, A, i, j) \simeq$ $\left(x^{\prime}, A^{\prime}, i^{\prime}, j^{\prime}\right)$ if $x=x^{\prime}$.

A semistandard notched bitableau $(P, Q)$ is said to be skew-symmetric if the following 2 conditions are satisfied simultaneously.

(i) The bitableau $(P, Q)$ should be of even size; that is, the number of elements in each row of $P$ and $Q$ should be even.

(ii) For any $(x, A, i, j),\left(x^{\prime}, A^{\prime}, i^{\prime}, j^{\prime}\right) \in \operatorname{set}(P, Q),(x, A$, $i, j) \preceq\left(x^{\prime}, A^{\prime}, i^{\prime}, j^{\prime}\right)$ if and only if $D_{(P, Q)}(x, A, i, j) \succeq$ $D_{(P, Q)}\left(x^{\prime}, A^{\prime}, i^{\prime}, j^{\prime}\right)$. Moreover, $(x, A, i, j) \prec\left(x^{\prime}, A^{\prime}, i^{\prime}\right.$, $\left.j^{\prime}\right)$ if and only if $D_{(P, Q)}(x, A, i, j) \quad>$

$$
\begin{aligned}
& D_{(P, Q)}\left(x^{\prime}, A^{\prime}, i^{\prime}, j^{\prime}\right) \text {, and }(x, A, i, j) \simeq\left(x^{\prime}, A^{\prime}, i^{\prime}, j^{\prime}\right) \text { if } \\
& \text { and only if } D_{(P, Q)}(x, A, i, j) \simeq D_{(P, Q)}\left(x^{\prime}, A^{\prime}, i^{\prime}, j^{\prime}\right) .
\end{aligned}
$$

Property (ii) will be henceforth referred to as the duality property associated to the skew-symmetric notched bitableau $(P, Q)$. Note that a skew-symmetric notched bitableau is a semistandard notched bitableau by default. The degree of a skew-symmetric notched bitableau $(P, Q)$ is the total number of boxes in $P$ (or $Q)$. The notions of negative, positive, and nonvanishing skew-symmetric notched bitableau remain the same as in Section 5 of [8]. The notion of a skew-symmetric notched bitableau $(P, Q)$ being bounded by $T, W$ (where $T, W$ are subsets of $\mathbb{N}^{2}$ ), and the notion of negative and positive parts of a skew-symmetric notched bitableau $(P, Q)$ remain the same as they were in Section 5 of [8].

If $(P, Q)$ is a nonvanishing skew-symmetric notched bitableau, define $\iota(P, Q)$ to be the notched bitableau obtained by reversing the order of the rows of $(Q, P)$. One checks that $\iota(P, Q)$ is a nonvanishing skew-symmetric notched bitableau. The map $\iota$ is an involution, and it maps negative skewsymmetric notched bitableaux to positive ones and vice versa. Thus ı gives a bijective pairing between the sets of negative and positive skew-symmetric notched bitableaux.

\section{Pairs of Skew-Symmetric Lexicographic Multisets on $\mathbb{N}^{2}$}

Recall the notions of a multiset on $\mathbb{N}^{2}$, finiteness of a multiset on $\mathbb{N}^{2}$ and degree of a multiset on $\mathbb{N}^{2}$, from Section 4 of [8]. By a lexicographic multiset on $\mathbb{N}^{2}$, we mean a finite multiset $\pi=\left\{\left(\alpha_{1}, \beta_{1}\right), \ldots,\left(\alpha_{t}, \beta_{t}\right)\right\}$ on $\mathbb{N}^{2}$ such that $\beta_{k} \geq \beta_{k+1} \forall k$, and if $\beta_{k}=\beta_{k+1}$, then $\alpha_{k} \geq \alpha_{k+1}, k=1, \ldots, t-1$. Given a lexicographic multiset $\pi$ on $\mathbb{N}^{2}$, let $\pi^{t}$ denote the multiset on $\mathbb{N}^{2}$ (which is not necessarily lexicographic) obtained by switching the two coordinates of $\pi$. We call the multiset $\pi^{t}$ on $\mathbb{N}^{2}$ the transpose of the multiset $\pi$. Consider a pair $\left\{\pi_{1}, \pi_{2}\right\}$ of multisets on $\mathbb{N}^{2}$ (not necessarily lexicographic), where both $\pi_{1}$ and $\pi_{2}$ are of the same degree (say, $t$ ). Let $\pi_{1}=\left\{\left(a_{1}, b_{1}\right), \ldots,\left(a_{t}, b_{t}\right)\right\}$, and $\pi_{2}=\left\{\left(d_{1}, c_{1}\right), \ldots,\left(d_{t}, c_{t}\right)\right\}$.

We call the first coordinate of the multiset $\pi_{1}$ the $a$-cell, the second coordinate of $\pi_{1}$ the $b$-cell, the first coordinate of $\pi_{2}$ the $d$-cell, and the second coordinate of $\pi_{2}$ the $c$-cell. Any entry in the pair $\left\{\pi_{1}, \pi_{2}\right\}$ of multisets on $\mathbb{N}^{2}$ can be identified uniquely by specifying 3 coordinates: the cell $\nabla$ of $\left\{\pi_{1}, \pi_{2}\right\}$ in which the entry lies $(\nabla=a, b, c$, or $d)$, the position $i$ (counting from left to right) of the entry in the cell $\nabla$, and the value $\sqsubseteq(\nabla, i)$ of the entry sitting in the $i$ th position of the cell $\nabla$. 
Set $S_{\pi_{1}, \pi_{2}}:=\{x \mid x=(\nabla, i, \sqsubseteq(\nabla, i)), \nabla \in\{a, b, c, d\}, i \in$ $\{1, \ldots, t\}\}$. For any $x \in S_{\pi_{1}, \pi_{2}}$, let

$$
\begin{aligned}
& D_{\pi_{1}, \pi_{2}}(x) \\
& :=\left\{\begin{array}{c}
(c, t+1-i, \sqsubseteq(c, t+1-i)) \\
\text { if } x=(a, i, \sqsubseteq(a, i)) \\
(d, t+1-i, \sqsubseteq(d, t+1-i)) \\
\text { if } x=(b, i, \sqsubseteq(b, i)) \\
(a, t+1-i, \sqsubseteq(a, t+1-i)) \\
\text { if } x=(c, i, \sqsubseteq(c, i)) \\
(b, t+1-i, \sqsubseteq(b, t+1-i)) \\
\text { if } x=(d, i, \sqsubseteq(d, i)) .
\end{array} \quad \forall i \in\{1, \ldots, t\}\right. \\
&
\end{aligned}
$$

We call $D_{\pi_{1}, \pi_{2}}(x)$ the dual of $x$ with respect to the pair $\left\{\pi_{1}, \pi_{2}\right\}$ of multisets on $\mathbb{N}^{2}$. Note that, for every $x \in S_{\pi_{1}, \pi_{2}}$, we have $D_{\pi_{1}, \pi_{2}}(x) \in S_{\pi_{1}, \pi_{2}}$. For any two elements $x, x^{\prime} \in S_{\pi_{1}, \pi_{2}}$, where $x=(\nabla, i, \sqsubseteq(\nabla, i))$ and $x^{\prime}=\left(\nabla^{\prime}, i^{\prime}, \sqsubseteq\left(\nabla^{\prime}, i^{\prime}\right)\right)$, we say that $x \precsim x^{\prime}$ if $\sqsubseteq(\nabla, i) \leq \sqsubseteq\left(\nabla^{\prime}, i^{\prime}\right)$. Similarly, we say that $x \precsim x^{\prime}$ if $\sqsubseteq(\nabla, i)<\sqsubseteq\left(\nabla^{\prime}, i^{\prime}\right)$ and $x \approx x^{\prime}$ if $\sqsubseteq(\nabla, i)=\sqsubseteq\left(\nabla^{\prime}, i^{\prime}\right)$.

The above pair $\left\{\pi_{1}, \pi_{2}\right\}$ of multisets on $\mathbb{N}^{2}$ is said to be skew-symmetric lexicographic if the following conditions are satisfied simultaneously.

(i) $\pi_{1}$ is a lexicographic multiset on $\mathbb{N}^{2}$.

(ii) $\pi_{2}^{t}$ is a lexicographic multiset on $\mathbb{N}^{2}$.

(iii) $a_{i}<d_{t+1-i} \forall i \in\{1, \ldots, t\}$.

(iv) $b_{i}<c_{t+1-i} \forall i \in\{1, \ldots, t\}$.

(v) For any $x, y \in S_{\pi_{1}, \pi_{2}}, x \lesssim y$ if and only if $D_{\pi_{1}, \pi_{2}}(x) \succsim D_{\pi_{1}, \pi_{2}}(y)$. Moreover, $x \precsim y$ if and only if $D_{\pi_{1}, \pi_{2}}(x) \succsim D_{\pi_{1}, \pi_{2}}(y)$, and $x \approx y^{\prime}$ if and only if $D_{\pi_{1}, \pi_{2}}(x) \approx D_{\pi_{1}, \pi_{2}}(y)(\rightarrow$ this property is called the duality property associated to the pair $\left\{\pi_{1}, \pi_{2}\right\}$ of skew-symmetric lexicographic multisets on $\mathbb{N}^{2}$ ).

For any pair $\left\{\pi_{1}, \pi_{2}\right\}$ of skew-symmetric lexicographic multisets on $\mathbb{N}^{2}$, we define the degree of the pair to be 2 times the degree of $\pi_{1}$ (or of $\pi_{2}$, they are the same). A pair $\left\{\pi_{1}, \pi_{2}\right\}$ of skew-symmetric lexicographic multisets on $\mathbb{N}^{2}$ is said to be negative if $a_{k}<b_{k}, k=1, \ldots, t$, positive if $a_{k}>b_{k}, k=$ $1, \ldots, t$, and nonempty if $a_{k} \neq b_{k}, k=1, \ldots, t$, where $\pi_{1}=$ $\left\{\left(a_{1}, b_{1}\right), \ldots,\left(a_{t}, b_{t}\right)\right\}$ and $\pi_{2}=\left\{\left(d_{1}, c_{1}\right), \ldots,\left(d_{t}, c_{t}\right)\right\}$. Note that condition (v) above will imply that if $a_{k}<b_{k} \forall k=1, \ldots, t$, then $d_{t+1-k}<c_{t+1-k} \forall k=1, \ldots, t$. Similarly, if $a_{k}>b_{k} \forall k=$ $1, \ldots, t$, then $d_{t+1-k}>c_{t+1-k} \forall k=1, \ldots, t$, and if $a_{k} \neq b_{k} \forall k=$ $1, \ldots, t$, then $d_{t+1-k} \neq c_{t+1-k} \forall k=1, \ldots, t$.

Let $\left\{\pi_{1}, \pi_{2}\right\}$ be a pair of nonempty skew-symmetric lexicographic multisets on $\mathbb{N}^{2}$ given by $\pi_{1}=\left\{\left(a_{1}, b_{1}\right), \ldots,\left(a_{t}, b_{t}\right)\right\}$ and $\pi_{2}=\left\{\left(d_{1}, c_{1}\right), \ldots,\left(d_{t}, c_{t}\right)\right\}$. Let us denote by $\pi_{1}^{-}$(resp., $\left.\pi_{1}^{+}\right)$ the lexicographic multiset on $\mathbb{N}^{2}$ consisting of those elements of $\pi_{1}$ such that $a_{i}<b_{i}$ (resp., $a_{i}>b_{i}$ ). Let us denote by $\pi_{2}^{-}$(resp., $\pi_{2}^{+}$) the lexicographic multiset on $\mathbb{N}^{2}$ consisting of those elements of $\pi_{2}$ such that $d_{i}<c_{i}$ (resp., $d_{i}>c_{i}$ ). We call $\left\{\pi_{1}^{-}, \pi_{2}^{-}\right\}$and $\left\{\pi_{1}^{+}, \pi_{2}^{+}\right\}$the negative and positive parts respectively of the pair $\left\{\pi_{1}, \pi_{2}\right\}$. Note here that because of condition (v) above, $\pi_{1}^{-}$and $\pi_{2}^{-}$will have the same degree, and the same holds true for $\pi_{1}^{+}$and $\pi_{2}^{+}$. It is easy to see now that both the pairs $\left\{\pi_{1}^{-}, \pi_{2}^{-}\right\}$and $\left\{\pi_{1}^{+}, \pi_{2}^{+}\right\}$of multisets on $\mathbb{N}^{2}$ are skew-symmetric lexicographic in their own right.

Given a lexicographic multiset $\pi$ on $\mathbb{N}^{2}$, define $l(\pi)$ to be the lexicographic multiset on $\mathbb{N}^{2}$ obtained by first switching the two coordinates of $\pi$ and then rearranging the elements so that the new multiset is lexicographic. Let $l^{t}$ be a map from the set of all lexicographic multisets on $\mathbb{N}^{2}$ to itself given by first switching the two coordinates of a given lexicographic multiset $\pi$ and then rearranging the elements so that the transpose of the resulting multiset becomes lexicographic.

We now define an involution $L$ on the set of all pairs of skew-symmetric lexicographic multisets on $\mathbb{N}^{2}$ by $L\left(\left\{\pi_{1}, \pi_{2}\right\}\right):=\left\{l\left(\pi_{1}\right), l^{t}\left(\pi_{2}\right)\right\}$. It is easy to check that this map is well defined, and it maps pairs of negative skew-symmetric lexicographic multisets on $\mathbb{N}^{2}$ to positive ones, and vice versa. Thus $L$ gives a bijective pairing between the set of all pairs of negative skew-symmetric lexicographic multisets on $\mathbb{N}^{2}$ and the set of all pairs of positive skew-symmetric lexicographic multisets on $\mathbb{N}^{2}$.

\section{The Orthogonal-Bounded- RSK Correspondence}

We next define the Orthogonal-bounded-RSK correspondence (OBRSK) as a function which maps a pair of negative skew-symmetric lexicographic multisets on $\mathbb{N}^{2}$ to a negative skew-symmetric notched bitableau. Let $\left\{\pi_{1}, \pi_{2}\right\}$ be a pair of negative skew-symmetric lexicographic multisets on $\mathbb{N}^{2}$ whose entries are labeled as in Section 5. We inductively form a sequence of notched bitableaux $\left(P^{(0)}, Q^{(0)}\right),\left(P^{(1)}, Q^{(1)}\right)$, $\ldots,\left(P^{(t)}, Q^{(t)}\right)$, such that each $\left(P^{(i)}, Q^{(i)}\right)$ is of even size, and $P^{(i)}$ is semistandard on $b_{i}$ for every $i=1, \ldots, t$ as follows.

Let $\left(P^{(0)}, Q^{(0)}\right)=(\emptyset, \emptyset)$, and let $b_{0}=b_{1}$. Assume inductively that we have formed $\left(P^{(i)}, Q^{(i)}\right)$, such that the notched bitableau $\left(P^{(i)}, Q^{(i)}\right)$ is of even size, $P^{(i)}$ is semistandard on $b_{i}$ and thus on $b_{i+1}$, since $b_{i+1} \leq b_{i}$.

Let us first fix some notation and terminology. Let $p_{k j}^{(i)}$ (resp., $q_{k j}^{(i)}$ ) denote the entry in the $k$ th row and $j$ th column of $P^{(i)}$ (resp., $Q^{(i)}$ ). Let $2 l_{k}^{(i)}$ denote the total number of entries (note that it is always even) in the $k$ th row of $P^{(i)}\left(\right.$ or $\left.Q^{(i)}\right)$.

Given an arbitrary notched tableau $P$ and any row number $k$ of $P$, we call the entry in the $j$ th box (counting from left to right) the forward jth entry of the kth row of $P$. Similarly, we call the entry in the $j$ th box (counting from right to left) of $P$ the backward jth entry of the kth row of $P$.

It is now easy to see that the backward $j$ th entry of the $k$ th row of $Q^{(i)}$ is actually equal to the forward $\left(2 l_{k}^{(i)}+1-j\right)$ th entry of $Q^{(i)}$. We now describe the OBRSK correspondence for the pair $\left\{\pi_{1}, \pi_{2}\right\}$ of negative skew-symmetric lexicographic multisets on $\mathbb{N}^{2}$ as mentioned above in Section 5.

Perform the bounded insertion process $P^{(i)} \stackrel{b_{i+1}}{\leftarrow} a_{i+1}$ as in [8]. In this finite-step process of bounded insertion, suppose that $a_{i+1}$ had bumped the "forward $j_{1}$ th entry" of the 1 st row of $P^{(i)<b_{i+1}}$ (see Section 3 of [8] for the notation $P^{(i)<b_{i+1}}$, it 
comes in the paragraph right after Example 3.1 of [8]); the "forward $j_{1}$ th entry" of the 1 st row of $P^{(i)<b_{i+1}}$ has bumped the "forward $j_{2}$ th entry" of the 2 nd row of $P^{(i)<b_{i+1}}, \ldots$ and so on until, at some point, a number is placed in a new box at the right end of some row of $P^{(i)<b_{i+1}}$, say this happens at the row number $K_{(i)}$ of $P^{(i)<b_{i+1}}$. Say that the entry of the new box (as mentioned in the previous statement) becomes the forward $j_{K_{(i)}}$ th entry of the $K_{(i)}$ th row of $P^{(i)} \stackrel{b_{i+1}}{\leftarrow} a_{i+1}$. We then construct a new notched tableau as follows.

We let $c_{t-i}$ bump the "backward $j_{1}$ th entry" of the 1 st row of $Q^{(i)}$; then we let the "backward $j_{1}$ th entry" of the 1 st row of $Q^{(i)}$ bump the "backward $j_{2}$ th entry" of the 2 nd row of $Q^{(i)}, \ldots$ and so on until, at some point, a number is placed in a new box at the backward $j_{K_{(i)}}$ th position of the $K_{(i)}$ th row of $Q^{(i)}$, shifting all entries in the backward 1 st... up to (and including) the backward $\left(j_{K_{(i)}}-1\right)$ th positions of the $K_{(i)}$ th row of $Q^{(i)}$ to the right by one box. Essentially, whatever we did for the bounded insertion process producing $P^{(i)} \stackrel{b_{i+1}}{\leftarrow} a_{i+1}$, we do a dual version of the same process on $Q^{(i)}$ with the integer $c_{t-i}$. We denote the resulting notched tableau by $Q^{(i)} \stackrel{\text { dual }}{\leftarrow} c_{t-i}$.

Note here that the tableaux $P^{(i)} \stackrel{b_{i+1}}{\leftarrow} a_{i+1}$ and $Q^{(i)} \stackrel{\text { dual }}{\leftarrow} c_{t-i}$ so constructed are of the same shape, but there exists one row in both of them in which the total number of entries is odd. We wanted to construct a notched bitableau $\left(P^{(i+1)}, Q^{(i+1)}\right)$ inductively from $\left(P^{(i)}, Q^{(i)}\right)$ which should be of even size. We make it possible in the following way.

Let $K_{(i)}$ be the row number of $P^{(i)}$ (or of $Q^{(i)}$ ) at which the above-mentioned insertion algorithm had stopped. Place $d_{t+1-(i+1)}\left(=d_{t-i}\right)$ in a new box at the rightmost end of the $K_{(i)}$ th row of $P^{(i)} \stackrel{b_{i+1}}{\leftarrow} a_{i+1}$. We denote the resulting notched tableau by $P^{(i+1)}$. It is an easy exercise to see that $P^{(i+1)}$ as constructed above will be semistandard on $b_{i+1}$. After this, we place $b_{i+1}$ in a new box at the leftmost end of the $K_{(i)}$ th row of $Q^{(i)} \stackrel{\text { dual }}{\leftarrow} c_{t-i}$, shifting all previously existing entries in the $K_{(i)}$ th row of $Q^{(i)} \stackrel{\text { dual }}{\leftarrow} c_{t-i}$ to the right by one box. We denote the resulting notched tableau by $Q^{(i+1)}$. Clearly $P^{(i+1)}$ and $Q^{(i+1)}$ have the same shape. Now we have got hold of a notched bitableau $\left(P^{(i+1)}, Q^{(i+1)}\right)$ which is of even size.

Then OBRSK $\left(\left\{\pi_{1}, \pi_{2}\right\}\right)$ is defined to be $\left(P^{(t)}, Q^{(t)}\right)$. In the process above, we write $\left(P^{(i+1)}, Q^{(i+1)}\right)=\left(P^{(i)}, Q^{(i)}\right) \stackrel{b_{i+1}, c_{t+1-(i+1)}}{\leftarrow}$ $a_{i+1}, d_{t+1-(i+1)}$. In terms of this notation, $\operatorname{OBRSK}\left(\left\{\pi_{1}, \pi_{2}\right\}\right)=$ $\left((\emptyset, \emptyset) \stackrel{b_{1}, c_{t}}{\leftarrow} a_{1}, d_{t}\right) \cdots \stackrel{b_{t}, c_{1}}{\leftarrow} a_{t}, d_{1}$.

Lemma 15. With notation as in the definition of the OBRSK correspondence mentioned above, $P^{(i)}$ is row strict for all $i \in$ $\{1, \ldots, t\}$.

Proof. We will prove the lemma by induction on $i$. The base case (i.e., when $i=1$ ) of induction is easy to see. Now let $i \in\{1, \ldots, t-1\}$. Assume inductively that $P^{(i)}$ is row strict.
We will now prove that $P^{(i+1)}$ is row strict. That $P^{(i)} \stackrel{b_{i+1}}{\longleftarrow} a_{i+1}$ is row strict follows in the same way as in [8]. Note that $P^{(i+1)}$ is obtained from $P^{(i)} \stackrel{b_{i+1}}{\leftarrow} a_{i+1}$ by adding $d_{t-i}$ at the rightmost end of some row of $P^{(i)} \stackrel{b_{i+1}}{\leftarrow} a_{i+1}$, say the $k$ th row. It now suffices to ensure that $d_{t-i}$ is strictly bigger than all entries in the $k$ th row of $P^{(i)} \stackrel{b_{i+1}}{\leftarrow} a_{i+1}$. It follows from the defining properties of the pair of negative skew-symmetric lexicographic multisets $\left\{\pi_{1}, \pi_{2}\right\}$ on $\mathbb{N}^{2}$ that $d_{t-i}$ is bigger than or equal to all entries of $P^{(i)} \stackrel{b_{i+1}}{\leftarrow} a_{i+1}$. But here we need to prove something sharper; namely, $d_{t-i}$ is strictly bigger than all entries in the $k$ th row of $P^{(i)} \stackrel{b_{i+1}}{\leftarrow} a_{i+1}$. We will prove this now.

Clearly all the entries of $P^{(i)} \stackrel{b_{i+1}}{\leftarrow} a_{i+1}$ are contained in $\left\{a_{1}, \ldots, a_{i+1}\right\} \dot{\cup}\left\{d_{t+1-i}, \ldots, d_{t}\right\}$ (see Section 4 of [8] for the notation $\dot{U})$. Also it is easy to observe that $a_{j}<d_{t-i} \forall j \in$ $\{1, \ldots, i+1\}$. So if the rightmost element of the $k$ th row of $P^{(i)} \stackrel{b_{i+1}}{\leftarrow} a_{i+1}$ equals $a_{j}$ for some $j \in\{1, \ldots, i+1\}$, then we are done. Otherwise, the element in the rightmost end of the $k$ th row of $P^{(i)} \stackrel{b_{i+1}}{\leftarrow} a_{i+1}$ is $d_{j}$ for some $j \in\{t+1-i, \ldots, t\}$ (say $j_{0}$ ). If $d_{j_{0}}<d_{t-i}$, then we are done. If not, then clearly $d_{j_{0}}=d_{t-i}$. It then follows from duality that $b_{t+1-j_{0}}=b_{i+1}$, and it is also clear that $t+1-j_{0}<i+1$.

But it is an easy exercise to check that if $l, l^{\prime} \in\{1, \ldots, t\}$ are such that $l<l^{\prime}$ and $b_{l}=b_{l^{\prime}}$, then the number of the row in which $d_{t+1-l^{\prime}}$ lies in $P^{\left(l^{\prime}\right)}$ is strictly bigger than the number of the row in which $d_{t+1-l}$ lies in $P^{\left(l^{\prime}\right)}$ (Here, the row number is counted from top to bottom.). So $d_{j_{0}}$ and $d_{t-i}$ cannot lie in the same row of $P^{(i+1)}$, a contradiction, hence proved.

The proof of the following lemma appears in Section 9.

Lemma 16. If $\left\{\pi_{1}, \pi_{2}\right\}$ is a pair of negative skew-symmetric lexicographic multisets on $\mathbb{N}^{2}$, then $\operatorname{OBRSK}\left(\left\{\pi_{1}, \pi_{2}\right\}\right)$ is a negative skew-symmetric notched bitableau.

Lemma 17. The map OBRSK is a degree-preserving bijection from the set of all pairs of negative skew-symmetric lexicographic multisets on $\mathbb{N}^{2}$ to the set of all negative skewsymmetric notched bitableaux.

Proof. That OBRSK is degree preserving is obvious. To show that OBRSK is a bijection, we define its inverse, which we call the reverse of OBRSK or ROBRSK.

Note that the entire procedure used to form $\left(P^{(i+1)}, Q^{(i+1)}\right)$ from $\left(P^{(i)}, Q^{(i)}\right), a_{i+1}, b_{i+1}, c_{t-i}$ and $d_{t-i}, i=1, \ldots, t-1$, is reversible. In other words, by knowing only $\left(P^{(i+1)}, Q^{(i+1)}\right)$, we can retrieve $\left(P^{(i)}, Q^{(i)}\right), a_{i+1}, b_{i+1}, c_{t-i}$, and $d_{t-i}$. First, we obtain $b_{i+1}$; it is the minimum entry of $Q^{(i+1)}$. Look at the lowest row in which $b_{i+1}$ appears in $Q^{(i+1)}$, say it is row number $s$ (counting from top to bottom). In the same row (row number $s$, counting from top to bottom) of $P^{(i+1)}$, look at the rightmost entry: this entry is precisely $d_{t-i}$. Remove this entry (which is $d_{t-i}$ ) from the sth row of $P^{(i+1)}$, that will give us the notched 
tableau $P^{(i)} \stackrel{b_{i+1}}{\leftarrow} a_{i+1}$. Similarly remove the leftmost entry (which is $b_{i+1}$ ) from the sth row of $Q^{(i+1)}$, and all other entries in this row of $Q^{(i+1)}$ should be moved one box to the left: this will give us the notched tableau $Q^{(i)} \stackrel{\text { dual }}{\leftarrow} c_{t-i}$.

Then, in the sth row of $P^{(i)} \stackrel{b_{i+1}}{\leftarrow} a_{i+1}$, select the greatest entry which is less than $b_{i+1}$. This entry was the new box of the bounded insertion. If we begin reverse bounded insertion with this entry, we retrieve $P^{(i)}$ and $a_{i+1}$. Look at the path in $P^{(i)} \stackrel{b_{i+1}}{\leftarrow} a_{i+1}$ starting from the $s$ th row to the topmost row, along which this reverse bounded insertion had happened. Trace the "dual path" in $Q^{(i)} \stackrel{\text { dual }}{\leftarrow} c_{t-i}$ and do a dual of the reverse bounded insertion (which was done originally on $P^{(i)} \stackrel{b_{i+1}}{\leftarrow} a_{i+1}$ to retrieve $P^{(i)}$ and $\left.a_{i+1}\right)$ on $Q^{(i)} \stackrel{\text { dual }}{\leftarrow} c_{t-i}$ : that will give us $Q^{(i)}$ and $c_{t-i}$ out of $Q^{(i)} \stackrel{\text { dual }}{\leftarrow} c_{t-i}$.

We call this process of obtaining $\left(P^{(i)}, Q^{(i)}\right), a_{i+1}, b_{i+1}$, $c_{t-i}$, and $d_{t-i}$ from $\left(P^{(i+1)}, Q^{(i+1)}\right)$ described in the paragraphs above a reverse step and denote it by $\left(P^{(i)}, Q^{(i)}\right)=$ $\left(P^{(i+1)}, Q^{(i+1)}\right) \stackrel{b_{i+1}, c_{-i}}{\longrightarrow} a_{i+1}, d_{t-i}$. We call the process of applying all the reverse steps sequentially to retrieve $\left\{\pi_{1}, \pi_{2}\right\}$ from $\left(P^{(t)}, Q^{(t)}\right)$ the reverse of OBRSK or ROBRSK.

If $\left(P^{(t)}, Q^{(t)}\right)$ is an arbitrary negative skew-symmetric notched bitableau (which we do not assume to be OBRSK $\left(\left\{\pi_{1}, \pi_{2}\right\}\right)$ for some $\left.\left\{\pi_{1}, \pi_{2}\right\}\right)$, then we can still apply a sequence of reverse steps to $\left(P^{(t)}, Q^{(t)}\right)$, to sequentially obtain $\left(P^{(i)}, Q^{(i)}\right), a_{i+1}, b_{i+1}, c_{t-i}$, and $d_{t-i}, i=t-1, \ldots, 1$. For this process to be well defined, however, it must first be checked that the successive $\left(P^{(i)}, Q^{(i)}\right)$ are negative skewsymmetric notched bitableaux. For this, it suffices to prove a statement very similar to that proved in Lemma 16; namely, "If $(P, Q)$ is a negative skew-symmetric notched bitableau, then $\left(P^{\prime}, Q^{\prime}\right):=(P, Q) \stackrel{b, c}{\longrightarrow} a, d$ is a negative skew-symmetric notched bitableau, $a<b, d<c, a<d, b<c$ are positive integers, $d$ is greater than or equal to all entries of $P$, and $b$ is less than or equal to all entries of $Q$." That $a<b, d<c, a<d$, and $b<c$ are positive integers, $d$ is greater than or equal to all entries of $P$, and $b$ is less than or equal to all entries of $Q$ follows immediately from the definition of a reverse step. That $\left(P^{\prime}, Q^{\prime}\right)$ is a negative skew-symmetric notched bitableau follows in much the same manner as the proof of Lemma 16; we omit the details.

It remains to be shown that the pair of multisets on $\mathbb{N}^{2}$ produced by applying this sequence of reverse steps to the arbitrary skew-symmetric notched bitableau $\left(P^{(t)}, Q^{(t)}\right)$ is skew-symmetric lexicographic. The proof of this uses the duality property of skew-symmetric notched bitableaux, the facts mentioned in the preceding paragraph regarding the integers $a, b, c$, and $d$, and the rest of the proof goes similarly as in the proof of Lemma 6.3 of [8].

At each step, OBRSK and the reverse of ROBRSK are inverse to each other. Thus they are inverse maps.

The map OBRSK can be extended to all pairs of nonempty skew-symmetric lexicographic multisets on $\mathbb{N}^{2}$.
If $\left\{\pi_{1}, \pi_{2}\right\}$ is a pair of positive skew-symmetric lexicographic multisets on $\mathbb{N}^{2}$, then define $\operatorname{OBRSK}\left(\left\{\pi_{1}, \pi_{2}\right\}\right)$ to be $\iota\left(\operatorname{OBRSK}\left(L\left(\left\{\pi_{1}, \pi_{2}\right\}\right)\right)\right)$, where the definition of the map $\iota$ is given at the end of Section 4. If $\left\{\pi_{1}, \pi_{2}\right\}$ is a pair of nonempty skew-symmetric lexicographic multisets on $\mathbb{N}^{2}$, with negative and positive parts $\left\{\pi_{1}^{-}, \pi_{2}^{-}\right\}$and $\left\{\pi_{1}^{+}, \pi_{2}^{+}\right\}$, then define $\operatorname{OBRSK}\left(\left\{\pi_{1}, \pi_{2}\right\}\right)$ to be the skew-symmetric notched bitableau whose negative and positive parts are OBRSK $\left(\left\{\pi_{1}^{-}, \pi_{2}^{-}\right\}\right)$and $\operatorname{OBRSK}\left(\left\{\pi_{1}^{+}, \pi_{2}^{+}\right\}\right)$.

Example 18. Let $\pi_{1}=\{(4,17),(3,17),(3,14),(7,10),(4,9)\}$, and $\pi_{2}=\{(20,25),(19,22),(15,26),(12,26),(12,25)\}$. See Figure 2.

Therefore.

$$
\operatorname{OBRSK}\left(\pi_{1}, \pi_{2}\right)=\left(\begin{array}{|l|l|l|l|l|l|l|l|l|l|}
\hline 3 & 4 & 12 & 19 \\
\hline 3 & 7 & 12 & 20 \\
\hline 4 & 15 & &
\end{array}, \begin{array}{|l|l|l|l|}
\hline 10 & 17 & 25 & 26 \\
\hline 9 & 17 & 22 & 26 \\
\hline 14 & 25 &
\end{array}\right) .
$$

\section{Restricting the OBRSK Correspondence}

7.1. Restricting by $\beta$. Let $\beta \in I(d)$. We say that a skewsymmetric notched bitableau $(P, Q)$ is on $\bar{\beta} \times \beta$, if all entries of $P$ are in $\bar{\beta}$, all entries of $Q$ are in $\beta$, and the sum of any entry in $P$ (or in $Q$ ) with its dual (with respect to $(P, Q)$ ) is $2 d+1$.

Given any monomial $U$ in $\mathfrak{D}(\beta)$, we can define a monomial $U^{\#}$ in $\mathfrak{A} \mathfrak{R}(\beta)$ as follows: $U^{\#}:=\left\{\left(c^{*}, r^{*}\right) \mid(r, c) \in U\right\}$. We say that a pair $\left\{V_{1}, V_{2}\right\}$ of skew-symmetric lexicographic multisets on $\mathbb{N}^{2}$ is a pair of skew-symmetric lexicographic multisets on $\bar{\beta} \times \beta$, if $V_{1}$ is a monomial in $\mathfrak{D} \mathfrak{R}(\beta), V_{2}$ is a monomial in $\mathfrak{A} \mathfrak{R}(\beta)$, a number of elements (counting multiplicities) in $V_{1}$ and $V_{2}$ are the same, and $V_{2}=V_{1}^{\#}$. It is easy to see from the construction of OBRSK that, if $\left\{U, U^{\#}\right\}$ is a pair of nonempty skew-symmetric lexicographic multisets on $\bar{\beta} \times \beta$, then OBRSK $\left(\left\{U, U^{\#}\right\}\right)$ is a nonvanishing skewsymmetric notched bitableau on $\bar{\beta} \times \beta$, and vice versa. Thus we obtain the following.

Corollary 19. The map OBRSK restricts to a degree-preserving bijection from the set of all pairs of nonempty (resp., negative, positive) skew-symmetric lexicographic multisets on $\bar{\beta} \times \beta$ to the set of all nonvanishing (resp., negative, positive) skewsymmetric notched bitableaux on $\bar{\beta} \times \beta$.

7.2. Restricting by $T$ and $W$. Let $T$ and $W$ be negative and positive subsets of $\mathbb{N}^{2}$, respectively, satisfying the condition that:

$$
T_{(1)}, T_{(2)}, W_{(1)} \text {, and } W_{(2)} \text { are subsets of } \mathbb{N} \text {, }
$$

where the notation $T_{(1)}, T_{(2)}, W_{(1)}$, and $W_{(2)}$ is explained in Section 4 of [8]. In this subsection, we will define the boundedness of a nonempty pair of skew-symmetric lexicographic multisets on $\mathbb{N}^{2}$ by $T, W$, and this is given in Definition 26, after some definitions which are necessary to understand it.

Definition 20. A dual pair of chains in $\mathbb{N}^{2}$ is a pair of subsets $\left\{C_{1}=\left\{\left(e_{1}, f_{1}\right), \ldots,\left(e_{m}, f_{m}\right)\right\}, C_{2}=\left\{\left(g_{1}, h_{1}\right), \ldots,\left(g_{m}, h_{m}\right)\right\}\right\}$ 


$$
\begin{aligned}
& P^{(0)}=\varnothing \\
& P^{(0)} \stackrel{14}{\leftarrow} 4=4 \\
& P^{(1)}=4 \begin{array}{l|l|}
4 & 12 \\
\hline
\end{array}
\end{aligned}
$$

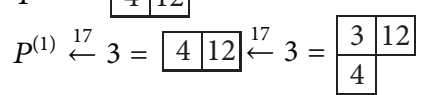

$$
\begin{aligned}
& P^{(2)}=\begin{array}{|l|l|}
\hline 3 & 12 \\
\hline 4 & 12 \\
\hline
\end{array} \\
& P^{(2)} \stackrel{14}{\leftarrow} 3=\begin{array}{|l|l|l|}
\hline 3 & 12 \\
\hline 4 & 12 \\
\hline
\end{array} \stackrel{14}{\leftarrow} 3=\begin{array}{|l|l|}
\hline 3 & 12 \\
\hline 3 & 12 \\
\hline 4 &
\end{array} \\
& P^{(3)}=\begin{array}{|l|l|}
\hline 3 & 12 \\
\hline 3 & 12 \\
\hline 4 & 15 \\
\hline
\end{array} \\
& P^{(3)} \stackrel{10}{\leftarrow} 7=\begin{array}{|l|l|}
\hline 3 & 12 \\
\hline 3 & 12 \\
\cline { 1 - 2 } 4 & 15 \\
\hline
\end{array} \stackrel{10}{\leftarrow} 7=\begin{array}{|l|l|l|}
\hline 3 & 7 & 12 \\
\hline 3 & 12 & \\
\hline 4 & 15 \\
\hline
\end{array} \\
& P^{(4)}=\begin{array}{|l|l|l|l|}
\hline 3 & 7 & 12 & 19 \\
\hline 3 & 12 & \multicolumn{1}{|c}{} \\
\cline { 1 - 2 } 4 & 15 & \multicolumn{1}{|l}{} \\
\hline
\end{array}
\end{aligned}
$$

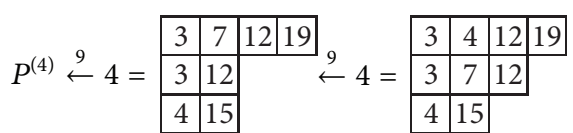

$$
\begin{aligned}
& P^{(5)}=\begin{array}{|c|c|c|c|}
\hline 3 & 4 & 12 & 19 \\
\hline 3 & 7 & 12 & 20 \\
\hline 4 & 15 & \multicolumn{3}{|l}{} \\
\hline
\end{array}
\end{aligned}
$$

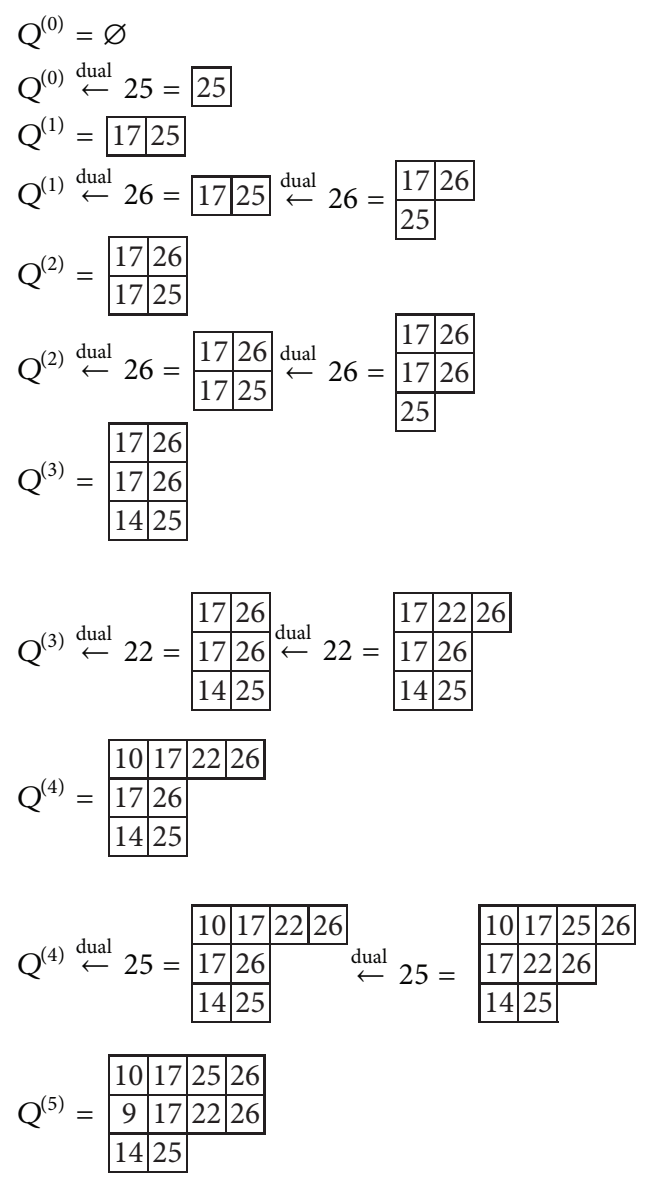

Figure 2

such that $C_{1}$ is a chain in $\mathbb{N}^{2}$ in the sense of Section 7 of [8], and $\left\{\sigma_{1}, \sigma_{2}\right\}$ is a pair of skew-symmetric lexicographic multisets on $\mathbb{N}^{2}$, where $\sigma_{1}=\left\{\left(e_{1}, f_{1}\right), \ldots,\left(e_{m}, f_{m}\right)\right\}$ and $\sigma_{2}=$ $\left\{\left(g_{1}, h_{1}\right), \ldots,\left(g_{m}, h_{m}\right)\right\}$.

Definition 21. Let $\left\{U_{1}, U_{2}\right\}$ be a pair of skew-symmetric lexicographic multisets on $\mathbb{N}^{2}$. Let $\left\{C_{1}, C_{2}\right\}$ be a dual pair of chains in $\mathbb{N}^{2}$ such that $C_{i}$ is contained in the underlying set of $U_{i}$ for all $i=1,2$. Given any element in $C_{1}$ (say, the $i$ th element counting from left to right), look at the first (counting from left to right) element in $U_{1}$ which is entrywise the same as the $i$ th element of $C_{1}$. Call this element of $U_{1}$ the $i_{\min }$ th element. Let $t$ be the total number of elements in $U_{1}$. One calls the $\left(t+1-i_{\text {min }}\right)$ th element of $U_{2}$ (counting from left to right) the dual element in $U_{2}$ corresponding to the $i$ th element of $C_{1}$.

Definition 22. Given any pair $\left\{\pi_{1}, \pi_{2}\right\}$ of skew-symmetric lexicographic multisets on $\mathbb{N}^{2}$, where $\pi_{1}=\left\{\left(a_{1}, b_{1}\right), \ldots,\left(a_{t}, b_{t}\right)\right\}$ and $\pi_{2}=\left\{\left(d_{1}, c_{1}\right), \ldots,\left(d_{t}, c_{t}\right)\right\}$, one says that the elements $\left(a_{i}, b_{i}\right)$ and $\left(d_{t+1-i}, c_{t+1-i}\right)$ of $\pi_{1}$ and $\pi_{2}$, respectively, are dual to each other with respect to $\left\{\pi_{1}, \pi_{2}\right\}$.

Definition 23. Given a pair of $\left\{U_{1}, U_{2}\right\}$ of skew-symmetric lexicographic multisets on $\mathbb{N}^{2}$, one says that a dual pair
$\left\{C_{1}, C_{2}\right\}$ of chains in $\mathbb{N}^{2}$ is a dual pair of chains in $\left\{U_{1}, U_{2}\right\}$, if the following two conditions are satisfied simultaneously: (i) $C_{i}$ is contained in the underlying set of $U_{i}$ for all $i=1,2$. (ii) Given any element of $C_{1}$ (say, the $i$ th element counting from left to right), the dual element in $U_{2}$ corresponding to it is entrywise the same as the dual element of the $i$ th element of $C_{1}$ with respect to $\left\{C_{1}, C_{2}\right\}$.

Definition 24. Given any row-strict notched bitableau $(P, Q)$, we associate with it 2 subsets of $\mathbb{N}^{2}$ as follows. Let $P_{1}$ and $Q_{1}$ denote the topmost rows of $P$ and $Q$, respectively. Let $p_{11}<\cdots<p_{1 k_{1}}$ and $q_{11}<\cdots<q_{1 k_{1}}$ denote the entries of $P_{1}$ and $Q_{1}$, respectively. We denote by $(P, Q)^{\text {up }}$ the subset of $\mathbb{N}^{2}$ given by $\left\{\left(p_{11}, q_{11}\right), \ldots,\left(p_{1 k_{1}}, q_{1 k_{1}}\right)\right\}$. We denote by $(P, Q)^{\text {down }}$ the subset of $\mathbb{N}^{2}$ obtained similarly if we work with the lowermost rows of $P$ and $Q$, instead of the topmost rows. See Example 25 for an illustration.

Example 25. Consider the row-strict notched bitableau

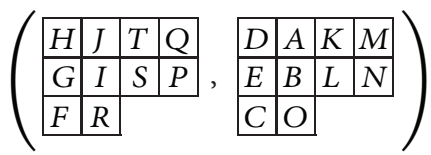


and call it $(\mathscr{P}, \mathcal{Q})$. Then $(\mathscr{P}, \mathcal{Q})^{\text {up }}=\{(H, D),(J, A),(T, K)$, $(Q, M)\}$, and $(\mathscr{P}, Q)^{\text {down }}=\{(F, C),(R, O)\}$.

Definition 26. A nonempty pair of skew-symmetric lexicographic multisets $\left\{U_{1}, U_{2}\right\}$ on $\mathbb{N}^{2}$ is said to be bounded by $T, W$ if for every dual pair $\left\{C_{1}, C_{2}\right\}$ of chains in $\left\{U_{1}, U_{2}\right\}$ one has

$$
\begin{gathered}
T \leq\left(P_{C_{1}^{-}, C_{2}^{-}}, Q_{C_{1}^{-}, C_{2}^{-}}\right)^{\text {up }}, \\
\left(P_{C_{1}^{+}, C_{2}^{+}}, Q_{C_{1}^{+}, C_{2}^{+}}\right)^{\text {down }} \leq W,
\end{gathered}
$$

where one uses the order on multisets on $\mathbb{N}^{2}$ defined in Section 4 of $[8] ;\left(P_{C_{1}^{-}, C_{2}^{-}}, Q_{C_{1}^{-}, C_{2}^{-}}\right)$is defined to be OBRSK $\left(\left\{C_{1}^{-}, C_{2}^{-}\right\}\right)$, and $\left(P_{C_{1}^{+}, C_{2}^{+}}, Q_{C_{1}^{+}, C_{2}^{+}}\right)$is defined as OBRSK $\left(\left\{C_{1}^{+}, C_{2}^{+}\right\}\right)$.

It is worthwhile to note that $\left(P_{C_{1}^{-}, C_{2}^{-}}, Q_{C_{1}^{-}, C_{2}^{-}}\right)^{\text {up }},\left(P_{C_{1}^{+}, C_{2}^{+}}\right.$, $\left.Q_{C_{1}^{+}, C_{2}^{+}}\right)^{\text {down }}, T$, and $W$ are subsets of $\mathbb{N}^{2}$, and they are not pairs of subsets!

With this definition, the OBRSK correspondence is a bounded function, in the sense that it maps bounded sets to bounded sets. More precisely, we have the following Lemma (to understand the statement of this lemma, we need to recall the notion of a semistandard notched bitableau being bounded by T, $W$ from Section 5 of [8]), whose proof appears in Section 9.

Lemma 27. If a pair $\left\{U_{1}, U_{2}\right\}$ of nonempty skew-symmetric lexicographic multisets on $\mathbb{N}^{2}$ is bounded by $T, W$, then $\operatorname{OBRSK}\left(\left\{U_{1}, U_{2}\right\}\right)$ is bounded by $T, W$.

Definition 28. A dual pair $\left\{C_{1}, C_{2}\right\}$ of chains in $\mathbb{N}^{2}$ is called a dual pair of chains in $\bar{\beta} \times \beta$ if $\left\{C_{1}, C_{2}\right\}$ is a pair of skewsymmetric lexicographic multisets on $\bar{\beta} \times \beta$.

Remark 29. A general dual pair of chains in $\bar{\beta} \times \beta$ will look like $\left\{C, C^{\#}\right\}$ for some extended $\beta$-chain $C$ in $\mathfrak{D} \mathfrak{R}(\beta)$. Note also that if $\left\{U_{1}, U_{2}\right\}$ is a pair of skew-symmetric lexicographic multisets on $\bar{\beta} \times \beta$, that is, if $U_{2}=U_{1}^{\#}$, then any dual pair of chains $\left\{C_{1}, C_{2}\right\}$ in $\left\{U_{1}, U_{2}\right\}$ must be a dual pair of chains in $\bar{\beta} \times \beta$; in other words, we must have $C_{2}=C_{1}^{\#}$, where $C_{1}$ is an extended $\beta$-chain in $\mathfrak{D} \mathfrak{R}(\beta)$.

Remark 30. Let $T$ and $W$ be negative and positive subsets of $\mathbb{N}^{2}$, respectively, satisfying (8). A nonempty pair of skewsymmetric lexicographic multisets $\left\{U, U^{\#}\right\}$ on $\bar{\beta} \times \beta$ is said to be bounded by $T, W$ if, for every dual pair of chains $\left\{C, C^{\#}\right\}$ in $\bar{\beta} \times \beta$ which is contained in the underlying set of $\left\{U, U^{\#}\right\}$,

$$
\begin{gathered}
T \leq\left(P_{C^{-}, C^{-\#}}, Q_{C^{-}, C^{-\#}}\right)^{\text {up }}, \\
\left(P_{C^{+}, C^{+}}, Q_{C^{+}, C^{+}}\right)^{\text {down }} \leq W,
\end{gathered}
$$

where we use the order on multisets on $\mathbb{N}^{2}$ defined in Section 4 of $[8]$, and $\left(P_{C^{-}, C^{-*}}, Q_{C^{-}, C^{-*}}\right)$ (resp., $\left(P_{C^{+}, C^{+*}}, Q_{C^{+}, C^{+}}\right)$) is defined to be OBRSK $\left(\left\{C^{-}, C^{-\#}\right\}\right.$ ) (resp., OBRSK
$\left.\left(\left\{C^{+}, C^{+\#}\right\}\right)\right)$. It is worthwhile to note that $\left(P_{C^{-}, C^{-\#}}, Q_{C^{-}, C^{-*}}\right)^{\text {up }}$, $\left(P_{C^{+}, C^{+}}, Q_{C^{+}, C^{+}}\right)^{\text {down }}, T$, and $W$ are subsets of $\mathbb{N}^{2}$; they are not pairs of subsets!

Let $T$ and $W$ be negative and positive subsets of $\bar{\beta} \times$ $\beta$, respectively, satisfying (8). Combining Corollary 19 and Lemma 27, we obtain the following.

Corollary 31. For any positive integer $m$, the number of pairs of nonempty skew-symmetric lexicographic multisets on $\bar{\beta} \times \beta$ bounded by $T, W$ of degree $2 m$ is less than or equal to the number of nonvanishing skew-symmetric notched bitableaux on $\bar{\beta} \times \beta$ bounded by $T, W$ of degree $2 m$.

\section{The Initial Ideal}

In this section, we will prove the main result of the paper, that is, Theorem 7.

Proof of Theorem 7. We wish to show that $\mathrm{in}_{\triangleright} I=$ $\left\langle\right.$ Chains $\left._{\alpha}^{\gamma}(\beta)\right\rangle$. Since we already know from Remark 12 that textChains ${ }_{\alpha}^{\gamma}(\beta) \subseteq \operatorname{in}_{\triangleright} I$, it follows that $\left\langle\right.$ Chains $\left._{\alpha}^{\gamma}(\beta)\right\rangle \subseteq \operatorname{in}_{\triangleright} I$. Hence it suffices to show that $\operatorname{in}_{\triangleright} I \subseteq\left\langle\right.$ Chains $\left._{\alpha}^{\gamma}(\beta)\right\rangle$ or equivalently that, for any $m \geq 1$, cardinality of the set of all degree $m$ monomials in $P /\left\langle\right.$ Chains $\left._{\alpha}^{\gamma}(\beta)\right\rangle$ is less than or equal to cardinality of the set of all degree $m$ monomials in $P /$ in $_{\triangleright} I$. Now since standard monomials on $Y_{\alpha, \beta}^{\gamma}$ (standard monomials on $Y_{\alpha, \beta}^{\gamma}$ is defined in Definition 32) and the monomials in $P /$ in $_{\triangleright} I$ both induce homogeneous bases for $P / I$, it suffices to show that, for any $m \geq 1$, cardinality of the set of all degree $m$ monomials in $P /\left\langle\right.$ Chains $\left._{\alpha}^{\gamma}(\beta)\right\rangle$ is less than or equal to cardinality of the set of all degree $m$ standard monomials on $Y_{\alpha, \beta}^{\gamma}$.

We wish to give a different indexing set for the standard monomials on $Y_{\alpha, \beta}^{\gamma}$. Let $I_{\beta}$ (skew-symm) denote the set of all pairs $(R, S)$ such that all of the following conditions are satisfied.

(i) $R \subset \bar{\beta}$.

(ii) $S \subset \beta$.

(iii) $|R|=|S|$, and this cardinality is even.

(iv) If $R=\left\{r_{1}<\cdots<r_{2 l}\right\}$ and $S=\left\{s_{1}<\cdots<s_{2 l}\right\}$, then $r_{i}+s_{2 l+1-i}=2 d+1 \forall i \in\{1, \ldots, 2 l\}$.

Defining $R-S:=R \dot{U}(\beta \backslash S)$ (see Section 4 of [8]), we have the following fact, which is easily verified.

The map $(R, S) \mapsto R-S$ is a bijection from $I_{\beta}$ (skewsymm) to $I(d)$, (Indeed, the inverse map is given by $\theta \mapsto$ $(\theta \backslash \beta, \beta \backslash \theta)$.).

Note that under this bijection, $(\emptyset, \emptyset)$ maps to $\beta$. Let $\left(R_{\alpha}, S_{\alpha}\right)$ and $\left(R_{\gamma}, S_{\gamma}\right)$ be the preimages of the elements $\alpha$ and $\gamma($ of $I(d))$, respectively. Define $T_{\alpha}$ and $W_{\gamma}$ to be any subsets of $\bar{\beta} \times \beta$ such that $\left(T_{\alpha}\right)_{(1)}=R_{\alpha},\left(T_{\alpha}\right)_{(2)}=S_{\alpha},\left(W_{\gamma}\right)_{(1)}=R_{\gamma}$, and $\left(W_{\gamma}\right)_{(2)}=S_{\gamma}$. Observe that $T_{\alpha}$ and $W_{\gamma}$ satisfy (8).

Under this identification of $I_{\beta}$ (skew-symm) with $I(d)$, the inequalities which define nonvanishing skew-symmetric notched bitableaux on $\bar{\beta} \times \beta$ bounded by $T_{\alpha}, W_{\gamma}$ are precisely 
the inequalities which define the standard monomials on $Y_{\alpha, \beta}^{\gamma}$ (the inequalities (12), (13), and (14) of this paper). Thus we obtain that the degree $2 m$ nonvanishing skew-symmetric notched bitableaux on $\bar{\beta} \times \beta$ bounded by $T_{\alpha}, W_{\gamma}$ forms an indexing set for the degree $m$ standard monomials on $Y_{\alpha, \beta}^{\gamma}$. Hence it suffices to show that, for any $m \geq 1$, cardinality of the set of all degree $m$ monomials in $P /\left\langle\right.$ Chains $\left._{\alpha}^{\gamma}(\beta)\right\rangle$ is less than or equal to cardinality of the set of all nonvanishing skewsymmetric notched bitableaux on $\bar{\beta} \times \beta$ bounded by $T_{\alpha}, W_{\gamma}$ of degree $2 m$.

On the other hand, it is easy to see that the set $\operatorname{Chains}_{\alpha}^{\gamma}(\beta)$ can be rewritten in the following format: Chains $\alpha_{\alpha}^{\gamma}(\beta)$ is the set of all $X_{C}$, where $C$ is any nonempty extended $\beta$-chain in $\mathfrak{D}(\beta)$ such that either (i)' or (ii)' below holds.

(i) $)^{\prime}$ The chain $C^{-}$is nonempty, and $R_{\alpha}-S_{\alpha} \not$ $\left(P_{C^{-}, C^{-*}}, Q_{C^{-}, C^{-\#}}\right)_{(1)}^{\text {up }}-\left(P_{C^{-}, C^{-\#}}, Q_{C^{-}, C^{-*}}\right)_{(2)}^{\text {up }}$.

(ii) ${ }^{\prime}$ The chain $C^{+}$is nonempty, and $\left(P_{C^{+}, C^{+\#}}, Q_{C^{+}, C^{+\#}}\right)_{(1)}^{\text {down }}-\left(P_{C^{+}, C^{+\#}}, Q_{C^{+}, C^{+\#}}\right)_{(2)}^{\text {down }} \nless$ $R_{\gamma}-S_{\gamma}$.

It is now obvious that the pairs of nonempty skewsymmetric lexicographic multisets on $\bar{\beta} \times \beta$ bounded by $T_{\alpha}, W_{\gamma}$ of degree $2 m$ form an indexing set for the degree $m$ monomials of $P /\left\langle\right.$ Chains $\left.{ }_{\alpha}^{\gamma}(\beta)\right\rangle$. Hence we have that, for any $m \geq 1$, cardinality of the set of all degree $m$ monomials in $P /\left\langle\right.$ Chains $\left._{\alpha}^{\gamma}(\beta)\right\rangle$ is equal to cardinality of the set of all pairs of nonempty skew-symmetric lexicographic multisets on $\bar{\beta} \times \beta$ bounded by $T_{\alpha}, W_{\gamma}$ of degree $2 m$.

Again from Corollary 31, it follows that for any $m \geq 1$, cardinality of the set of all pairs of nonempty skew-symmetric lexicographic multisets on $\bar{\beta} \times \beta$ bounded by $T_{\alpha}, W_{\gamma}$ of degree $2 m$ is less than or equal to cardinality of the set of all nonvanishing skew-symmetric notched bitableaux on $\bar{\beta} \times$ $\beta$ bounded by $T_{\alpha}, W_{\gamma}$ of degree $2 m$. Thus $\left\langle\right.$ Chains $\left.s_{\alpha}^{\gamma}(\beta)\right\rangle \supseteq$ $\operatorname{in}_{\triangleright} I$.

Definition 32. Let $P=\mathfrak{E}\left[X_{(r, c)} \mid(r, c) \in \mathfrak{D} \mathfrak{R}(\beta)\right]$. Recall the concept of a Pfaffian (denoted by $f_{\theta, \beta}$ for $\theta \in I(d)$ ) from Section 3.3 of this paper. One calls $f=f_{\theta_{1}, \beta} \cdots f_{\theta_{r}, \beta} \in P$ a standard monomial if $\theta_{1}, \ldots, \theta_{r} \in I(d)$,

$$
\theta_{1} \leq \cdots \leq \theta_{r}
$$

and for each $i \in\{1, \ldots, r\}$, either

$$
\theta_{i}<\beta \quad \text { or } \theta_{i}>\beta \text {. }
$$

If in addition, for $\alpha, \gamma \in I(d)$,

$$
\alpha \leq \theta_{1}, \quad \theta_{r} \leq \gamma,
$$

then we say that $f$ is standard on $Y_{\alpha, \beta}^{\gamma}$. We define the degree of the standard monomial $f_{\theta_{1}, \beta} \cdots f_{\theta_{r}, \beta}$ to be the sum of the $\beta$-degrees of $\theta_{1}, \ldots, \theta_{r}$, where for any $\theta \in I(d)$; the $\beta$-degree is defined to be one-half the cardinality of $\theta \backslash \beta$.

Remark 33. In general, a standard monomial is not a monomial in the affine coordinates $X_{(r, c)},(r, c) \in \mathfrak{D} \mathfrak{R}(\beta)$; rather, it is a polynomial. It is only a monomial in the $f_{\theta, \beta}$ 's.

\section{Proofs}

In this section, we give proofs of Lemmas 16 and 27. To give a proof of Lemma 16, we need Lemma 34 mentioned below whose proof is left to the reader as an exercise.

Lemma 34. If $A$ and $B$ are two finite multisets on $\mathbb{N}$ of the same degree such that $A \leq B$ (where the termwise order $\leq$ on multisets on $\mathbb{N}$ and the degree of a multiset on $\mathbb{N}$ are as defined in Section 4 of [8]) and $p, q$ are natural numbers such that $p \leq$ $q$, then $A \dot{\cup}\{p\} \leq B \dot{\cup}\{q\}$.

\subsection{Proof of Lemma 16}

Proof. The proof is by induction. The base case of induction is easy to see, and hence we omit the details. Let $\left\{\pi_{1}^{(t-1)}, \pi_{2}^{(t-1)}\right\}$ be a pair of negative skew-symmetric lexicographic multisets on $\mathbb{N}^{2}$, where $\pi_{1}^{(t-1)}=\left\{\left(a_{1}, b_{1}\right), \ldots,\left(a_{t-1}, b_{t-1}\right)\right\}$ and $\pi_{2}^{(t-1)}=$ $\left\{\left(d_{2}, c_{2}\right), \ldots,\left(d_{t}, c_{t}\right)\right\}$. Let $(P, Q)=\operatorname{OBRSK}\left(\left\{\pi_{1}^{(t-1)}, \pi_{2}^{(t-1)}\right\}\right)$. Assume inductively that $(P, Q)$ is a negative skew-symmetric notched bitableau. Now let $\left\{\pi_{1}^{(t)}, \pi_{2}^{(t)}\right\}$ be a pair of negative skew-symmetric lexicographic multisets on $\mathbb{N}^{2}$ such that $\pi_{1}^{(t)}=\pi_{1}$ and $\pi_{2}^{(t)}=\pi_{2}$, where $\pi_{1}$ and $\pi_{2}$ are given by $\pi_{1}=\left\{\left(a_{1}, b_{1}\right), \ldots,\left(a_{t}, b_{t}\right)\right\}$ and $\pi_{2}=\left\{\left(d_{1}, c_{1}\right), \ldots,\left(d_{t}, c_{t}\right)\right\}$. Let $\left(P^{\prime}, Q^{\prime}\right)=\operatorname{OBRSK}\left(\left\{\pi_{1}^{(t)}, \pi_{2}^{(t)}\right\}\right)$. It suffices to show that $\left(P^{\prime}, Q^{\prime}\right)$ is also a negative skew-symmetric notched bitableau.

We will first prove that $\left(P^{\prime}, Q^{\prime}\right)$ is a negative semistandard notched bitableau. The fact that $P^{\prime}$ is row strict follows from Lemma 15. It then follows from duality that $Q^{\prime}$ is also row strict. Hence $\left(P^{\prime}, Q^{\prime}\right)$ is row strict. Let $r^{\prime}$ be the total number of rows of $P^{\prime}\left(\right.$ or $\left.Q^{\prime}\right)$. Let $P_{i}^{\prime}, Q_{i}^{\prime}, P_{i}$, and $Q_{i}$ denote the multiset consisting of all elements in the $i$ th row of $P^{\prime}, Q^{\prime}, P$, and $Q$, respectively. In fact, these multisets $\left(P_{i}^{\prime}, Q_{i}^{\prime}, P_{i}, Q_{i}\right)$ happen to be sets because $P^{\prime}, Q^{\prime}, P$, and $Q$ are row strict. It needs to be shown that $P_{i}^{\prime}-Q_{i}^{\prime} \leq P_{i+1}^{\prime}-Q_{i+1}^{\prime}$ for $1 \leq i \leq r^{\prime}-1$, and $P_{r^{\prime}}^{\prime}-Q_{r^{\prime}}^{\prime} \lessdot \emptyset$ (see Section 4 of [8] for the notation $\lessdot$ ). We will prove the former statement first. To prove that $P_{i}^{\prime}-Q_{i}^{\prime} \leq$ $P_{i+1}^{\prime}-Q_{i+1}^{\prime}$ for $1 \leq i \leq r^{\prime}-1$, there will be three nontrivial possibilities which will be registered below as Cases 1,2 , and 3 , respectively.

Case 1. $a_{t}$ and $d_{1}$ are added to the first row of $P$, and $c_{1}$ and $b_{t}$ are added to the first row of $Q$. All rows other than the 1 st row of $P$ and $Q$ remain unchanged.

In this case, it suffices to show that $P_{1} \dot{\cup} Q_{2} \dot{\cup}\left\{a_{t}, d_{1}\right\} \leq$ $P_{2} \cup \dot{\cup} Q_{1} \dot{\cup}\left\{c_{1}, b_{t}\right\}$. It follows from induction hypothesis that $P_{1} \dot{\cup} Q_{2} \leq P_{2} \dot{\cup} Q_{1}$. Since $\left\{\pi_{1}^{(t)}, \pi_{2}^{(t)}\right\}$ is a pair of negative skew-symmetric lexicographic multisets on $\mathbb{N}^{2}$, we have $a_{t}<$ $b_{t}$ and $d_{1}<c_{1}$. Therefore $a_{t} \leq b_{t}$ and $d_{1} \leq c_{1}$. These facts together with Lemma 34 (applied twice) imply easily that $P_{1} \dot{\cup} Q_{2} \dot{\cup}\left\{a_{t}, d_{1}\right\} \leq P_{2} \dot{\cup} Q_{1} \dot{\cup}\left\{c_{1}, b_{t}\right\}$. Hence we are done in this case.

Case 2. $x_{p}$ bumps $y_{p}$ from $P_{i}, y_{p}$ bumps $z_{p}$ from $P_{i+1}$, and the dual bumping happens on $Q_{i}$ and $Q_{i+1}$. Let us express the dual bumping by saying that $x_{q}$ bumps $y_{q}$ from $Q_{i}$ and $y_{q}$ bumps $z_{q}$ from $Q_{i+1}$. 
Clearly then, $x_{p} \leq y_{p} \leq z_{p}<b_{t}$, and hence $x_{q} \geq y_{q} \geq$ $z_{q}$ by the property (ii) in the definition of a skew-symmetric notched bitableau. Again since all entries in $Q$ are $\geq b_{t}$, we get that $x_{q} \geq y_{q} \geq z_{q} \geq b_{t}$.

We need to show that $P_{i}^{\prime}-Q_{i}^{\prime} \leq P_{i+1}^{\prime}-Q_{i+1}^{\prime}$, or, in other words, $P_{i}^{\prime} \dot{\cup} Q_{i+1}^{\prime} \leq P_{i+1}^{\prime} \dot{\cup} Q_{i}^{\prime}$. Note that $P_{i}^{\prime} \dot{\cup} Q_{i+1}^{\prime}=$ $\left[\left(P_{i} \dot{\cup} Q_{i+1}\right) \backslash\left\{y_{p}, z_{q}\right\}\right] \dot{\cup}\left\{x_{p}, y_{q}\right\}$ and $P_{i+1}^{\prime} \dot{\cup} Q_{i}^{\prime}=\left[\left(P_{i+1} \dot{\cup} Q_{i}\right) \backslash\right.$ $\left.\left\{z_{p}, y_{q}\right\}\right] \dot{\cup}\left\{x_{q}, y_{p}\right\}$. It suffices to show that $\left(P_{i} \dot{\cup} Q_{i+1}\right) \backslash$ $\left\{y_{p}, z_{q}\right\} \leq\left(P_{i+1} \dot{\cup} Q_{i}\right) \backslash\left\{z_{p}, y_{q}\right\}$ because if we can prove this much, then since $x_{p} \leq y_{p}$ and $x_{q} \geq y_{q}$ we can apply Lemma 34 and get done with the proof as in Case 1 .

We will now prove that $\left(P_{i} \dot{\cup} \mathrm{Q}_{i+1}\right) \backslash\left\{y_{p}, z_{q}\right\} \leq\left(P_{i+1} \dot{\cup}\right.$ $\left.Q_{i}\right) \backslash\left\{z_{p}, y_{q}\right\}$. Since $z_{p}<b_{t}$ and all entries of $Q_{i}$ are $\geq b_{t}$, therefore $z_{p}$ is the smallest element in $P_{i+1} \cup \dot{U} Q_{i}$ which is $\geq y_{p}$. It then follows from duality that $z_{q}$ is the biggest element in $P_{i} \dot{\cup} Q_{i+1}$, that is, $\leq y_{q}$. Also since $y_{p}<b_{t} \leq z_{q}$, we have $y_{p}<z_{q}$, and hence by duality $y_{q}>z_{p}$. Also by induction hypothesis, $P_{i} \dot{\cup} Q_{i+1} \leq P_{i+1} \dot{\cup} Q_{i}$. All these facts put together prove the required thing easily, and we are done in this case.

Case 3. $x_{p}$ bumps $y_{p}$ from $P_{i}$, and $y_{p}$ along with $d_{1}$ are added to $P_{i+1}$. The dual phenomenon happens with $Q_{i}$ and $Q_{i+1}$; we express the dual phenomenon by saying that $x_{q}$ bumps $y_{q}$ from $Q_{i}$, and $y_{q}$ along with $b_{t}$ are added to $Q_{i+1}$.

Clearly then, $x_{p} \leq y_{p}<b_{t}$, and hence $x_{q} \geq y_{q}$ by the property (ii) in the definition of a Skew-symmetric notched bitableau. Again since all entries in $Q$ are $\geq b_{t}$, we get that $x_{q} \geq y_{q} \geq b_{t}$. Also $y_{p}<d_{1}$ since the position of $d_{1}$ is at the rightmost end of that row of $P_{i+1}$ in which $y_{p}$ is placed. Hence by duality, we have $y_{q}>b_{t}$.

We need to show that $P_{i}^{\prime}-Q_{i}^{\prime} \leq P_{i+1}^{\prime}-Q_{i+1}^{\prime}$, or, in other words, $P_{i}^{\prime} \dot{\cup} Q_{i+1}^{\prime} \leq P_{i+1}^{\prime} \dot{\cup} Q_{i}^{\prime}$. Note that $P_{i}^{\prime} \dot{\cup} Q_{i+1}^{\prime}=$ $\left(P_{i} \dot{\cup} Q_{i+1} \backslash\left\{y_{p}\right\}\right) \dot{\cup}\left\{b_{t}\right\} \dot{\cup}\left\{x_{p}, y_{q}\right\}$, and $P_{i+1}^{\prime} \dot{\cup} Q_{i}^{\prime}=\left(P_{i+1} \dot{U}\right.$ $\left.Q_{i} \backslash\left\{y_{q}\right\}\right) \dot{U}\left\{d_{1}\right\} \dot{\cup}\left\{x_{q}, y_{p}\right\}$. Using Lemma 34, it is enough to prove that $\left(P_{i} \dot{\cup} Q_{i+1} \backslash\left\{y_{p}\right\}\right) \dot{\cup}\left\{b_{t}\right\} \leq\left(P_{i+1} \dot{\cup} Q_{i} \backslash\left\{y_{q}\right\}\right) \dot{\cup}\left\{d_{1}\right\}$, which we will do now.

Let $P_{i} \cup \dot{\cup} Q_{i+1}$ be given by $X_{1} \leq \cdots \leq X_{2 n}$, and let $P_{i+1} \cup \dot{\cup} Q_{i}$ be given by $Y_{1} \leq \cdots \leq Y_{2 n}$ (since the bitableau $(P, Q)$ is of even size, the cardinality of $P_{i} \dot{\cup} Q_{i+1}$ or of $P_{i+1} \cup \dot{ } Q_{i}$ is even). Note that there are no elements of $P_{i+1}$ which are $\geq y_{p}$ and $<b_{t}$. Also since $b_{t}$ is $\leq$ all elements of $Q_{i}$, therefore we can conclude that there are no elements of $P_{i+1} \cup \dot{\cup} Q_{i}$ which are $\geq y_{p}$ and $<b_{t}$. It follows from duality that there are no elements of $P_{i} \dot{\cup} Q_{i+1}$ which are $\leq y_{q}$ as well as $>d_{1}$. Let $\left(P_{i} \dot{\cup} Q_{i+1} \backslash\left\{y_{p}\right\}\right) \dot{\cup}\left\{b_{t}\right\}$ be given by

$$
\begin{aligned}
X_{1} & \leq \cdots \leq X_{i-1} \leq X_{i+1} \leq \cdots \leq X_{j} \\
& \leq b_{t} \leq X_{j+1} \leq \cdots \leq X_{2 n}
\end{aligned}
$$

and let $\left(P_{i+1} \dot{\cup} Q_{i} \backslash\left\{y_{q}\right\}\right) \dot{\cup}\left\{d_{1}\right\}$ be given by

$$
\begin{aligned}
Y_{1} & \leq \cdots \leq Y_{2 n-j} \leq d_{1} \leq Y_{2 n-j+1} \leq \cdots \leq Y_{2 n-i} \\
& \leq Y_{2 n-i+2} \leq \cdots \leq Y_{2 n} .
\end{aligned}
$$

This means that $y_{p}=X_{i}$ and its dual $y_{\mathrm{q}}=Y_{2 n-i+1}$ (the $i$ th element counting from right to left in the ordered sequence
$\left.Y_{1} \leq \cdots \leq Y_{2 n}\right)$. Also since $y_{p}=X_{i}$ and $y_{p}<b_{t}$, it follows that $i<j$. Observe that $b_{t}$ is the $j$ th element (counting from left to right) in the ordered sequence $X_{1} \leq \cdots \leq X_{i-1} \leq X_{i+1} \leq$ $\cdots \leq X_{j} \leq b_{t} \leq X_{j+1} \leq \cdots \leq X_{2 n}$ of integers and $d_{1}$ (the dual of $b_{t}$ with respect to the pair $\left\{\pi_{1}, \pi_{2}\right\}$ of multisets) is the $j$ th element (counting from right to left) in the ordered sequence $Y_{1} \leq \cdots \leq Y_{2 n-j} \leq d_{1} \leq Y_{2 n-j+1} \leq \cdots \leq Y_{2 n-i} \leq Y_{2 n-i+2} \leq$ $\cdots \leq Y_{2 n}$ of integers. Consider any element in $\left(P_{i} \dot{\cup} Q_{i+1} \mid\right.$ $\left.\left\{y_{p}\right\}\right) \dot{\cup}\left\{b_{t}\right\}$. It can either be $X_{k}$ for some $k \neq i$ or $b_{t}$. If it is $X_{k}$ for some $k \neq i$, then we either need to show that $X_{k} \leq Y_{k}$, $X_{k} \leq d_{1}$, or $X_{k} \leq Y_{k-1}$ (these are the only 3 possibilities; the last possibility $X_{k} \leq Y_{k-1}$ happens if and only if $k \in\{2 n-j+$ $2, \ldots, 2 n-i+1\})$. If it is $b_{t}$, then we need to show that $b_{t} \leq$ the $j$ th element (counting from left to right) of $\left(P_{i+1} \dot{\cup} Q_{i} \backslash\right.$ $\left.\left\{y_{q}\right\}\right) \dot{\cup}\left\{d_{1}\right\}$, which can either be $Y_{j}, d_{1}$, or $Y_{j-1}$.

After all these observation(s), the proof follows easily using the inequalities $i<j, x_{p} \leq y_{p}<b_{t}, x_{q} \geq y_{q} \geq b_{t}$, $y_{p}<d_{1}$, and $y_{q}>b_{t}$ and the facts that there are no elements of $P_{i+1} \cup \dot{\cup} Q_{i}$ which lie in the interval $\left[y_{p}, b_{t}\right)$ and that there are no elements of $P_{i} \cup \dot{Q} Q_{i+1}$ which lie in the interval $\left(d_{1}, y_{q}\right]$.

We have now proved that $\left(P^{\prime}, Q^{\prime}\right)$ is a semistandard notched bitableau. To prove that the semistandard notched bitableau $\left(P^{\prime}, Q^{\prime}\right)$ is negative, it is enough to prove that $P_{r^{\prime}}^{\prime}-$ $Q_{r^{\prime}}^{\prime} \lessdot \emptyset$, where $r^{\prime}$ denotes the total number of rows of $P^{\prime}\left(\right.$ or $\left.Q^{\prime}\right)$. If $P_{r^{\prime}}^{\prime}=P_{r^{\prime}}$ and $Q_{r^{\prime}}^{\prime}=Q_{r^{\prime}}$, then since $(P, Q)$ is assumed to be a negative skew-symmetric notched bitableau, it follows immediately that $P_{r^{\prime}}^{\prime}-Q_{r^{\prime}}^{\prime} \lessdot \emptyset$. Otherwise, we do the following: let $P_{r^{\prime}}^{\prime}$ and $Q_{r^{\prime}}^{\prime}$ be given by $\lambda_{1}<\cdots<\lambda_{s^{\prime}}$ and $\delta_{1}<\cdots<\delta_{s^{\prime}}$, respectively. It follows easily from the way the OBRSK algorithm works that there exists at least one entry in $P_{r^{\prime}}^{\prime}$ which is $<b_{t}$. Let $\lambda_{l}$ denote the largest entry in $P_{r^{\prime}}^{\prime}$ which is $<b_{t}$. Since $b_{t} \leq \delta_{1}$, it now follows easily that $\lambda_{j}<\delta_{j} \forall j \in\{1, \ldots, l\}$. Since $\lambda_{l}$ is the largest entry in $P_{r^{\prime}}^{\prime}$ which is $\left\langle b_{t}\right.$, therefore $l \geq 1$, and it follows from duality that $\delta_{s^{\prime}+1-l}=\delta_{s^{\prime}-(l-1)}>d_{1}$. Hence $\delta_{s^{\prime}} \geq \delta_{s^{\prime}-(l-1)}>d_{1}=\lambda_{s^{\prime}}$. It only remains to show that if $l+1<s^{\prime}$, then $\lambda_{j}<\delta_{j} \forall j \in$ $\left\{l+1, \ldots, s^{\prime}-1\right\}$. Clearly since $l \geq 1$ and $l+1<s^{\prime}$, it follows that, in this case, the last row of $P$ (resp., $Q$ ) is the $r^{\prime}$ th row, namely $P_{r^{\prime}}$ (resp., $Q_{r^{\prime}}$ ), and $\delta_{s^{\prime}-(l-1)}$ is the new box of the dual insertion in $Q_{r^{\prime}}$. Since $\lambda_{s^{\prime}}=d_{1}, \delta_{s^{\prime}-(l-1)}>d_{1}, P_{r^{\prime}}-Q_{r^{\prime}} \lessdot \emptyset$ by induction hypothesis and $\lambda_{j}<\lambda_{s^{\prime}} \forall j \in\left\{l+1, \ldots, s^{\prime}-1\right\}$, it is now easy to see that $\lambda_{j}<\delta_{j} \forall j \in\left\{l+1, \ldots, s^{\prime}-1\right\}$.

Hence we have proved that $\left(P^{\prime}, Q^{\prime}\right)$ is a negative semistandard notched bitableau. The fact that $\left(P^{\prime}, Q^{\prime}\right)$ is skewsymmetric is easy to see from the very construction of the OBRSK algorithm and from the fact that the pair $\left\{\pi_{1}^{(t)}, \pi_{2}^{(t)}\right\}$ of multisets is skew-symmetric, lexicographic.

\subsection{Proof of Lemma 27}

Proof (proof of Lemma 27). Let $T$ be a negative subset of $\mathbb{N}^{2}$ such that $T_{(1)}, T_{(2)}$ are subsets of $\mathbb{N}$. Suppose that $\left\{U_{1}=\right.$ $\left.\left\{\left(a_{1}, b_{1}\right), \ldots,\left(a_{t}, b_{t}\right)\right\}, U_{2}=\left\{\left(d_{1}, c_{1}\right), \ldots,\left(d_{t}, c_{t}\right)\right\}\right\}$ is a pair of negative skew-symmetric lexicographic multisets on $\mathbb{N}^{2}$. For each $m=1, \ldots, t$, let $U_{1}^{(m)}:=\left\{\left(a_{1}, b_{1}\right), \ldots,\left(a_{m}, b_{m}\right)\right\}$ and $U_{2}^{(m)}:=\left\{\left(d_{t+1-m}, c_{t+1-m}\right), \ldots,\left(d_{t}, c_{t}\right)\right\}$. Let $\left(P^{(m)}, Q^{(m)}\right):=$ 
$\operatorname{OBRSK}\left(\left\{U_{1}^{(m)}, U_{2}^{(m)}\right\}\right)$. For proving Lemma 27, it suffices to show that if $\left\{U_{1}, U_{2}\right\}$ is bounded by $T, \emptyset$, then $\left(P^{(m)}, Q^{(m)}\right)$ is bounded by $T, \emptyset$ for all $m=1, \ldots, t$ because then the proof of Lemma 27 will follow similarly as the proofs of parts (iii), (iv), and (v) of Lemma 11.1 in [8].

Assume that $\left\{U_{1}, U_{2}\right\}$ is bounded by $T, \emptyset$. For every $m \in$ $\{1, \ldots, t\}$, let $P_{1}^{(m)}$ (resp., $Q_{1}^{(m)}$ ) denote the topmost row of $P^{(m)}$ (resp., $Q^{(m)}$ ). We need to show that $T_{(1)}-T_{(2)} \leq P_{1}^{(m)}-Q_{1}^{(m)}$. Equivalently, we need to show that for all positive integers $z,\left|\left(T_{(1)}-T_{(2)}\right)^{\leq z}\right| \geq\left|\left(P_{1}^{(m)}-Q_{1}^{(m)}\right)^{\leq z}\right|$, where we use the definition $A-B:=A \dot{U}(\mathbb{N} \backslash B)$ for any two subsets $A$ and $B$ of $\mathbb{N}$ (see Section 4 of [8]). We will prove this by induction on $m$. Let us first see the base case of induction. When $m=1$, $U_{1}^{(m)}=\left\{\left(a_{1}, b_{1}\right)\right\}$ and $U_{2}^{(m)}=\left\{\left(d_{t}, c_{t}\right)\right\}$. Let $C_{1}=\left\{\left(a_{1}, b_{1}\right)\right\}$ and $C_{2}=\left\{\left(d_{t}, c_{t}\right)\right\}$. Since $\left\{U_{1}, U_{2}\right\}$ is bounded by $T, \emptyset$, it follows that for the dual pair $\left\{C_{1}, C_{2}\right\}$ of chains in $\left\{U_{1}, U_{2}\right\}$, we have $T \leq\left(P_{C_{1}^{-}, C_{2}^{-}}, Q_{C_{1}^{-}, C_{2}^{-}}\right)^{\text {up }} \leq \emptyset$. But it can be easily seen that $\left(P_{C_{1}^{-}, C_{2}^{-}}, Q_{C_{1}^{-}, C_{2}^{-}}\right)^{\text {up }}=\left\{\left(a_{1}, b_{1}\right),\left(d_{t}, c_{t}\right)\right\}$. This proves the base case of induction. Fix an integer $k \in\{1, \ldots, t-1\}$, and assume that $\left(P^{(m)}, Q^{(m)}\right)$ is bounded by $T, \emptyset$ for all $m \leq k$. We will now show that $\left(P^{(k+1)}, Q^{(k+1)}\right)$ is bounded by $T, \emptyset$.

Before we begin the proof, let us fix some notation for the rest of this subsection. Define $\left\{p_{1}^{(k)}, \ldots, p_{2 c_{k}}^{(k)}\right\}$ to be the topmost row of $P^{(k)}$ and $\left\{q_{1}^{(k)}, \ldots, q_{2 c_{k}}^{(k)}\right\}$ the topmost row of $Q^{(k)}$, both listed in increasing order. Let $(P, Q)=\left(P^{(k)}\right.$, $\left.Q^{(k)}\right), a=a_{k+1}, b=b_{k+1}, c=c_{t-k}, d=d_{t-k}$, $\left(P^{\prime}, Q^{\prime}\right)=\left(P^{(k+1)}, Q^{(k+1)}\right),\left\{p_{1}, \ldots, p_{2 \widehat{c}}\right\}=\left\{p_{1}^{(k)}, \ldots, p_{2 c_{k}}^{(k)}\right\}$, and $\left\{q_{1}, \ldots, q_{2 \widehat{c}}\right\}=\left\{q_{1}^{(k)}, \ldots, q_{2 c_{k}}^{(k)}\right\}$. Note that $\left\{p_{1}, \ldots, p_{2 \widehat{c}}\right\} \subset\left\{a_{1}\right.$, $\left.\ldots, a_{k}\right\} \dot{\cup}\left\{d_{t+1-k}, \ldots, d_{t}\right\}$ and $\left\{q_{1}, \ldots, q_{2 \widehat{c}}\right\} \subset\left\{b_{1}, \ldots, b_{k}\right\} \dot{\cup}$ $\left\{c_{t+1-k}, \ldots, c_{t}\right\}$. Let $P_{1}$ (resp., $Q_{1}$ ) denote the topmost row of $P$ (resp., Q). Similarly let $P_{1}^{\prime}$ (resp., $Q_{1}^{\prime}$ ) denote the topmost row of $P^{\prime}$ (resp., $Q^{\prime}$ ). We consider two cases corresponding to the two ways in which $\left(P_{1}^{\prime}, Q_{1}^{\prime}\right)$ can be obtained from $\left(P_{1}, Q_{1}\right)$.

Case 1. $P_{1}^{\prime}$ is obtained by a bumping $p_{l}$ in $P_{1}$, for some $1 \leq$ $l \leq 2 \widehat{c}$, that is, $P_{1}^{\prime}=\left(P_{1} \backslash\left\{p_{l}\right\}\right) \dot{\cup}\{a\}$ and $Q_{1}^{\prime}=\left(Q_{1} \backslash\right.$ $\left.\left\{q_{2 \widehat{c}+1-l}\right\}\right) \dot{\cup}\{c\}$.

Since $b$ is less than or equal to all elements of $\left\{b_{1}, \ldots, b_{k}\right\}$ and $b_{i}<c_{t+1-i} \forall i \in\{1, \ldots, t\}$, it follows that $b \leq$ all elements of $\left\{b_{1}, \ldots, b_{k}\right\} \dot{\cup}\left\{c_{t+1-k}, \ldots, c_{t}\right\}$. Therefore $a<b \leq q_{1}$, and hence by duality $c>d \geq p_{2 \widehat{c}}$. The fact that $a$ bumps $p_{l}$ implies that $a \leq p_{l}$ and $p_{l}<b$. Hence $a \leq p_{l}<b \leq q_{1}$, and therefore by duality, we have $c \geq q_{2 \widehat{c}+1-l}>d \geq p_{2 \widehat{c}}$. For $z<a, p_{l} \leq z<$ $q_{2 \widehat{c}+1-l}$, or $z \geq c$,

$$
\begin{aligned}
\left|\left(T_{(1)}-T_{(2)}\right)^{\leq z}\right| & \geq\left|\left(P_{1}-Q_{1}\right)^{\leq z}\right| \\
& =\left|\left(P_{1}^{\prime}-Q_{1}^{\prime}\right)^{\leq z}\right|,
\end{aligned}
$$

where the first inequality follows from induction hypothesis, and second equality follows from the facts that $p_{l-1}<a \leq p_{l}<b \leq q_{1} \leq q_{2 \widehat{c}+1-l} \leq c$ and $p_{2 \widehat{c}} \leq d<c$. If $a=$ $p_{l}$, then we are done. Thus we assume that $a<p_{l}$ (and hence by duality that $\left.c>q_{2 \widehat{c}+1-l}\right)$. We now need to consider only two possible positions of $z$, namely, $a \leq z<p_{l}$ and $q_{2 \hat{c}+1-l} \leq z<c$. We claim that, for $z$ such that $a \leq z<p_{l}$ or $q_{2 \widehat{c}+1-l} \leq z<c$, there exists a dual pair of chains $\left\{C_{k+1}^{(1)}, C_{k+1}^{(2)}\right\}$ in $\left\{U_{1}^{(k+1)}, U_{2}^{(k+1)}\right\}$ such that $\left|\left(\left(P_{C_{k+1}^{(1)-}, C_{k+1}^{(2)-}}, Q_{C_{k+1}^{(1)-}, C_{k+1}^{(2)-}}\right)_{(1)}^{\text {up }}-\left(P_{C_{k+1}^{(1)-},} C_{k+1}^{(2)-}, Q_{C_{k+1}^{(1)-},}, C_{k+1}^{(2)-}\right)_{(2)}^{\text {up }}\right)^{\leq z}\right| \geq$ $\left|\left(P_{1}^{\prime}-Q_{1}^{\prime}\right)^{\leq z}\right|$. Assuming the claim and using the fact that $T \leq\left(P_{C_{k+1}^{(1)-}, C_{k+1}^{(2)-}}, Q_{C_{k+1}^{(1)-}}, C_{k+1}^{(2)-}\right)^{\text {up }}$ (which is because $\left\{U_{1}, U_{2}\right\}$ is bounded by $T, \emptyset)$, we are done. The claim follows from Lemmas 41 and 42 .

Case 2. $P_{1}^{\prime}$ is obtained by adding $a$ to $P_{1}$ in position $l$ from the left and adding $d$ to $P_{1}$ at the rightmost end (after $p_{2 \widehat{c}}$ ); $Q_{1}^{\prime}$ is obtained from $Q_{1}$ by adding $b$ to the leftmost end of $Q_{1}$ and adding $c$ at the backward $l$ th position of $Q_{1}$. That is, $P_{1}^{\prime}=$ $P_{1} \dot{\cup}\{a, d\}=\left\{p_{1}<\cdots<p_{l-1}<a<p_{l}<\cdots<p_{2 \widehat{c}}<d\right\}$, and $Q_{1}^{\prime}=Q_{1} \dot{\cup}\{b, c\}=\left\{b<q_{1}<\cdots<q_{2 \widehat{c}+1-l}<c<q_{2 \widehat{c}+1-(l-1)}<\right.$ $\left.\cdots<q_{2 \hat{c}}\right\}$.

Note that $p_{l-1}<a<b<q_{1}, a<b \leq p_{l}$ (since $b>$ $p_{l}$ would require that $a$ bumps $p_{l}$ in the bounded insertion process), and $a<b<d<c$. For $z<a$,

$$
\left|\left(T_{(1)}-T_{(2)}\right)^{\leq z}\right| \geq\left|\left(P_{1}-Q_{1}\right)^{\leq z}\right|=\left|\left(P_{1}^{\prime}-Q_{1}^{\prime}\right)^{\leq z}\right|,
$$

where the first inequality follows from induction hypothesis and the second equality follows from the facts that $p_{l-1}<a<$ $p_{l}$ and $a<b<q_{1}$. For $z$ such that $b \leq z<d$, note that

$$
\begin{aligned}
\left|\left(P_{1}^{\prime}-Q_{1}^{\prime}\right)^{\leq z}\right| & =\left|\left(P_{1}^{\prime} \dot{U}\left(\mathbb{N} \backslash Q_{1}^{\prime}\right)\right)^{\leq z}\right| \\
& =\left|\left(P_{1}^{\prime}\right)^{\leq z}\right|+\left|\left(\mathbb{N} \backslash Q_{1}^{\prime}\right)^{\leq z}\right| \\
& =\left(\left|\left(P_{1}\right)^{\leq z}\right|+1\right)+\left(\left|\left(\mathbb{N} \backslash Q_{1}\right)^{\leq z}\right|-1\right) \\
& =\left|\left(P_{1}\right)^{\leq z}\right|+\left|\left(\mathbb{N} \backslash Q_{1}\right)^{\leq z}\right| \\
& =\left|\left(P_{1}-Q_{1}\right)^{\leq z}\right| .
\end{aligned}
$$

Hence for $b \leq z<d$, we have

$$
\left|\left(T_{(1)}-T_{(2)}\right)^{\leq z}\right| \geq\left|\left(P_{1}-Q_{1}\right)^{\leq z}\right|=\left|\left(P_{1}^{\prime}-Q_{1}^{\prime}\right)^{\leq z}\right|
$$

For $z \geq c$,

$$
\begin{aligned}
\left|\left(P_{1}^{\prime}-Q_{1}^{\prime}\right)^{\leq z}\right| & =\left|\left(P_{1}^{\prime} \dot{U}\left(\mathbb{N} \backslash Q_{1}^{\prime}\right)\right)^{\leq z}\right| \\
& =\left|\left(P_{1}^{\prime}\right)^{\leq z}\right|+\left|\left(\mathbb{N} \backslash Q_{1}^{\prime}\right)^{\leq z}\right| \\
& =\left(\left|\left(P_{1}\right)^{\leq z}\right|+2\right)+\left(\left|\left(\mathbb{N} \backslash Q_{1}\right)^{\leq z}\right|-2\right) \\
& =\left|\left(P_{1}\right)^{\leq z}\right|+\left|\left(\mathbb{N} \backslash Q_{1}\right)^{\leq z}\right| \\
& =\left|\left(P_{1}-Q_{1}\right)^{\leq z}\right| .
\end{aligned}
$$

Hence for $z \geq c$, we have

$$
\left|\left(T_{(1)}-T_{(2)}\right)^{\leq z}\right| \geq\left|\left(P_{1}-Q_{1}\right)^{\leq z}\right|=\left|\left(P_{1}^{\prime}-Q_{1}^{\prime}\right)^{\leq z}\right|
$$


It now remains to show that for $z$ such that $a \leq z<b$ or $d \leq z<c$,

$$
\left|\left(T_{(1)}-T_{(2)}\right)^{\leq z}\right| \geq\left|\left(P_{1}^{\prime}-Q_{1}^{\prime}\right)^{\leq z}\right| .
$$

We claim that for $z$ such that $a \leq z<b$ or $d \leq z<c$, there exists a dual pair of chains $\left\{S_{k+1}^{(1)}, S_{k+1}^{(2)}\right\}$ in $\left\{U_{1}^{(k+1)}, U_{2}^{(k+1)}\right\}$ such that

$$
\begin{aligned}
& \mid\left(\left\{\text { topmost row of } P_{S_{k+1}^{(1)}} S_{k+1}^{(2)}\right\}\right. \\
& \left.\quad \dot{U}\left(\mathbb{N} \backslash\left\{\text { topmost row of } Q_{S_{k+1}^{(1)}, S_{k+1}^{(2)}}\right\}\right)\right)^{\leq z} \mid \\
& \quad \geq\left|\left(P_{1}^{\prime}-Q_{1}^{\prime}\right)^{\leq z}\right| .
\end{aligned}
$$

Assuming the claim, we are done as in Case 1. But the proof of the claim is similar to the proof of the claim made in Case 1 , so we omit it.

Hence we are done with the required proof modulo, the proof of the claim made in Case 1. As mentioned in Case 1, the claim follows from Lemmas 41 and 42 .

It now remains to prove Lemmas 41 and 42 . But before we go to prove these two lemmas, let us have a close look at their statements and try to understand the reason behind generating such complicated lemmas for the purpose of proving the claim. It is clear that once we understand the reason behind the statement of Lemma 41 being so complicated, Lemma 42 is a natural thing to be proved for finishing the proof of the claim. Observe that Lemma 41 looks like a Generalization of Lemma 11.1 (i) of [8] in the present case of the Orthogonal grassmannian. In Lemma 11.1 (i) of [8], Kreiman says that, for $1 \leq j \leq m(k)$, there exists a chain $C_{k, j}$ in $U^{(k)}$ which has $j$ elements, the last of which has first component $p_{j}^{(k)}$. We need to compare this statement of Kreiman with the statement of Lemma 41 here. The fact that we need a dual pair of chains here (in Lemma 41) instead of "a chain" (as mentioned in Lemma 11.1 (i) of [8]) is quite natural to believe. But it is not apparently clear as to why we need the dual pair of chains in Lemma 41 to satisfy a "strange" condition which is condition (\#\#) (this condition is explained in Definition 40). We will now illustrate by an example (see Example 35 and the illustration associated to it below) that asking for the dual pair of chains in Lemma 41 to satisfy condition (\#\#) is not at all strange, in fact it is the only natural thing to be asked for in the present case of the Orthogonal grassmannian. Another explanation for the same is provided in Remark 36.

Example 35. Let $d=5$ and $\beta=(5,7,8,9,10)$. Consider the multiset $U=\{(1,9),(3,7)\}$ in $\mathbb{N}^{2}$ contained in the underlying set of $\bar{\beta} \times \beta$. Applying the map BRSK (which is defined in Section 6 of [8]) to the multiset $U$, we get BRSK $(U)=$ $\left(\begin{array}{|l|l|l|}\hline 1 & 3 \\ \hline & 9 & 9\end{array}\right)$. Set $C_{1,1}=C_{2,1}=\{(1,9)\}$ and $C_{2,2}=U$. It is easy to see that the chains $C_{1,1}, C_{2,1}$, and $C_{2,2}$ satisfy the required conditions of lemma 11.1 (i) of [8].

On the other hand, let us take the pair of negative skewsymmetric lexicographic multisets on $\mathbb{N}^{2}$ given by $U_{1}, U_{2}$, where $U_{1}=U$ and $U_{2}=U^{\#}=\{(4,8),(2,10)\}$. Observe that OBRSK $\left(\left\{U, U^{\#}\right\}\right)=\left(\begin{array}{l|l|l|l|}1 & 2 & 3 & 4 \\ \hline\end{array}\right.$ $X=10$ (we have denoted 10 by the alphabet $X$ here because the young tableau package used here does not work with 2digit integers). Set $C_{1,1}^{(1)}=\{(1,9)\}, C_{1,1}^{(2)}=\{(2,10)\}, C_{1,2}^{(1)}=$ $\{(1,9)\}, C_{1,2}^{(2)}=\{(2,10)\}, C_{2,1}^{(1)}=\{(1,9)\}, C_{2,1}^{(2)}=\{(2,10)\}$, $C_{2,2}^{(1)}=\{(1,9)\}, C_{2,2}^{(2)}=\{(2,10)\}, C_{2,3}^{(1)}=U_{1}, C_{2,3}^{(2)}=U_{2}$, and $C_{2,4}^{(1)}=U_{1}, C_{2,4}^{(2)}=U_{2}$. It is easy to see that according to the notation of Lemma 41 , we have $m(k)=2$ for $k=1$ and $m(k)=4$ for $k=2$. It can also be easily verified that, for each $k \in\{1,2\}$ and each $j \in\{1, \ldots, m(k)\}$, the dual pair $\left\{C_{k, j}^{(1)}, C_{k, j}^{(2)}\right\}$ of chains (as mentioned above) satisfies the required condition (which is condition (\#\#) with respect to the bitableau $\left(P^{(k)}, Q^{(k)}\right)$ and the integer $\left.j\right)$ of Lemma 41. In fact, more is true; these dual pairs of chains satisfy condition (\#\#)-strong (this condition is explained in Definition 39) with respect to the bitableau $\left(P^{(k)}, Q^{(k)}\right)$ and the integer $j$ for every $k \in\{1,2\}$ and $j \in\{1, \ldots, m(k)\}$.

Illustration Associated to Example 35. A possible analog of Lemma 11.1 (i) of [8] in the present case of the Orthogonal grassmannian would be to demand for the dual pair of chains $\left\{C_{k, j}^{(1)}, C_{k, j}^{(2)}\right\}$ in Lemma 41 to have exactly $j$ elements in $C_{k, j}^{(1)}$ (as well as in $C_{k, j}^{(2)}$ ) and the last element of $C_{k, j}^{(1)}$ to have first coordinate $p_{j}^{(k)}$. But it is easy to see from Example 35 that such a demand is meaningless. Instead, it would be natural and meaningful to demand for the dual pair $\left\{C_{k, j}^{(1)}, C_{k, j}^{(2)}\right\}$ of chains in Lemma 41 to satisfy condition (\#\#)-strong (this is introduced in Definition 39) with respect to the bitableau $\left(P^{(k)}, Q^{(k)}\right)$ and the integer $j$. But in Lemma 41, we demand the dual pair of chains $\left\{C_{k, j}^{(1)}, C_{k, j}^{(2)}\right\}$ to satisfy condition (\#\#) (this is introduced in Definition 40) which is little weaker a condition to be satisfied than condition (\#\#)-strong. The reason behind this is that if we demand for the dual pair $\left\{C_{k, j}^{(1)}, C_{k, j}^{(2)}\right\}$ of chains to satisfy condition (\#\#)-strong instead of condition (\#\#), then the proof of Lemma 41 becomes more difficult. This difficulty (in the proof of Lemma 41) arises at the point(s) where we need to show that the dual pair $\left\{C_{k+1, l}^{(1)}, C_{k+1, l}^{(2)}\right\}$ of chains in $\left\{U_{1}^{(k+1)}, U_{2}^{(k+1)}\right\}$ satisfies condition (\#\#)-strong. It is easier to show that the dual pair $\left\{C_{k+1, l}^{(1)}, C_{k+1, l}^{(2)}\right\}$ of chains satisfies condition (\#\#). This completes the explanation behind the statement of Lemma 41 being so complicated.

Remark 36. Note that the properties of the dual pair $\left\{C_{k, j}^{(1)}, C_{k, j}^{(2)}\right\}$ of chains in Lemma 41 are a Generalization of the properties of the chain $C_{k, j}$ of Lemma 11.1 of [8]. In fact, this Generalization precisely reveals the difference between the OBRSK algorithm (when applied to a dual pair of chains) in the present case of the Orthogonal Grassmannian and the BRSK algorithm of [8] (when applied to a chain) in the case of the ordinary Grassmannian. Equivalently, this Generalization reveals the difference between the concept of " $\mathfrak{D}$-domination of a chain by an element of $I(d)$ " in the case 
of the Orthogonal Grassmannian (as in Section 2.4.4 of [9]) and the concept of "domination of a chain by an element of $I(d, 2 d)$ " in the case of the ordinary Grassmannian as in [6].

Let us now prove Lemmas 41 and 42, but for the proofs we need some definitions which are the following.

Definition 37. Let $(P, Q)$ and $k_{1}$ be as in Definition 24. Let $j \in\left\{1, \ldots, k_{1}\right\}$. With notation as in Definition 24 , one calls $\left(p_{1 j}, q_{1 j}\right)$ the jth element of $(P, Q)^{\mathrm{up}}$, and we denote by $(P, Q)_{\leq j}^{\text {up }}$ the subset $\left\{\left(p_{11}, q_{11}\right), \ldots,\left(p_{1 j}, q_{1 j}\right)\right\}$ of $(P, Q)^{\text {up }}$. See Example 38 for an illustration.

Example 38. Consider the row-strict notched bitableau $(\mathscr{P}, Q)$ of Example 25. If we take $j=3$, then the $j$ th element of $(\mathscr{P}, Q))^{\text {up }}$ is $(T, K)$ and $(\mathscr{P}, Q)_{\leq j}^{\text {up }}$ equals $\{(H, D),(J, A),(T, K)\}$. We can work out similarly for other possible values of $j$.

Definition 39. Let $(P, Q)$ be a negative skew-symmetric notched bitableau. Let $\left\{p_{1}, \ldots, p_{2 c}\right\}$ and $\left\{q_{1}, \ldots, q_{2 c}\right\}$ be the topmost row of $P$ and $Q$, respectively, both listed in increasing order. Let $j \in\{1, \ldots, 2 c\}$. We say that a dual pair $\left\{C_{1}, C_{2}\right\}$ of chains in $\mathbb{N}^{2}$ satisfies condition (\#\#)-strong with respect to the bitableau $(P, Q)$ and the integer $j$ if all the following conditions are satisfied simultaneously.

(i) $\left|C_{1}\right|,\left|C_{2}\right| \leq j$.

(ii) The first coordinate of the $j$ th element of $\left(P_{C_{1}^{-}, C_{2}^{-}}\right.$, $\left.Q_{C_{1}^{-}, C_{2}^{-}}\right)^{\text {up }}$ is $p_{j}$.

(iii) All the entries which occur as first coordinates of elements of $C_{1}$ form a subset of the set of all entries which occur as first coordinates of elements of $\left(P_{C_{1}^{-}, C_{2}^{-}}, Q_{C_{1}^{-}, C_{2}^{-}}\right)_{\leq j}^{\text {up }}$.

Definition 40. Let $(P, Q)$ be a negative skew-symmetric notched bitableau. Let $\left\{p_{1}, \ldots, p_{2 c}\right\}$ and $\left\{q_{1}, \ldots, q_{2 c}\right\}$ be the topmost row of $P$ and $Q$, respectively, both listed in increasing order. Let $j \in\{1, \ldots, 2 c\}$. One says that a dual pair $\left\{C_{1}, C_{2}\right\}$ of chains in $\mathbb{N}^{2}$ satisfies condition (\#\#) with respect to the bitableau $(P, Q)$ and the integer $j$ if all the following conditions are satisfied simultaneously.

(i) $\left|C_{1}\right|,\left|C_{2}\right| \leq j$.

(ii) There exists an integer $\chi_{j}$ which is $\geq j$ such that the first coordinate of the $\chi_{j}$ th element of $\left(P_{C_{1}^{-}, C_{2}^{-}}, Q_{C_{1}^{-}, C_{2}^{-}}\right)^{\text {up }}$ is $p_{j}$.

(iii) All the entries which occur as first coordinates of elements of $C_{1}$ form a subset of the set of all entries which occur as first coordinates of elements of $\left(P_{C_{1}^{-}, C_{2}^{-}}, Q_{C_{1}^{-}, C_{2}^{-}}\right)_{\leq \chi_{j}}^{\text {up }}$.

Lemma 41. Let $\left\{U_{1}=\left\{\left(a_{1}, b_{1}\right), \ldots,\left(a_{t}, b_{t}\right)\right\}, U_{2}=\left\{\left(d_{1}, c_{1}\right)\right.\right.$, $\left.\left.\ldots,\left(d_{t}, c_{t}\right)\right\}\right\}$ be a pair of negative skew-symmetric lexicographic multisets on $\mathbb{N}^{2}$. For $k=1, \ldots, t$, let $U_{1}^{(k)}:=$ $\left\{\left(a_{1}, b_{1}\right), \ldots,\left(a_{k}, b_{k}\right)\right\}$ and $U_{2}^{(k)}:=\left\{\left(d_{t+1-k}, c_{t+1-k}\right), \ldots,\left(d_{t}, c_{t}\right)\right\}$. Let $\left(P^{(k)}, Q^{(k)}\right):=\operatorname{OBRSK}\left(\left\{U_{1}^{(k)}, U_{2}^{(k)}\right\}\right)$. Define $\left\{p_{1}^{(k)}, \ldots, p_{2 c_{k}}^{(k)}\right\}$ to be the topmost row of $P^{(k)}$ and $\left\{q_{1}^{(k)}, \ldots, q_{2 c_{k}}^{(k)}\right\}$ the topmost row of $Q^{(k)}$, both listed in increasing order. Let $m(k)$ := $\max \left\{m \in\left\{1, \ldots, 2 c_{k}\right\} \mid p_{m}^{(k)}<q_{1}^{(k)}\right\}=\left|\left(P_{1}^{(k)}\right)^{<q_{1}^{(k)}}\right|$. Then for $1 \leq j \leq m(k)$, there exists a dual pair of chains $\left\{C_{k, j}^{(1)}, C_{k, j}^{(2)}\right\}$ in $\left\{U_{1}^{(k)}, U_{2}^{(k)}\right\}$ which satisfies condition (\#\#) with respect to the bitableau $\left(P^{(k)}, Q^{(k)}\right)$ and the integer $j$. Let one denote by $\chi_{k, j}$ the integer corresponding to condition (\#\#) (item number (ii), which is $\geq j$ ).

Proof. We prove this by induction on $k$, with $k=1$ the starting point of induction. When $k=1, U_{1}^{(1)}=\left\{\left(a_{1}, b_{1}\right)\right\}$ and $U_{2}^{(1)}=\left\{\left(d_{t}, c_{t}\right)\right\}$. There are two possible cases, namely, when $m(k)=1$ and $m(k)=2$. In both the cases, for every $j \in\{1, \ldots, m(k)\}$, take $C_{1, j}^{(1)}=\left\{\left(a_{1}, b_{1}\right)\right\}$ and $C_{1, j}^{(2)}=\left\{\left(d_{t}, c_{t}\right)\right\}$. This proves the base case of induction.

Let $(P, Q)=\left(P^{(k)}, Q^{(k)}\right), a=a_{k+1}, b=b_{k+1}, c=$ $c_{t-k}, d=d_{t-k},\left(P^{\prime}, Q^{\prime}\right)=\left(P^{(k+1)}, Q^{(k+1)}\right),\left\{p_{1}, \ldots, p_{2 \widehat{c}}\right\}=$ $\left\{p_{1}^{(k)}, \ldots, p_{2 c_{k}}^{(k)}\right\}$, and $\left\{q_{1}, \ldots, q_{2 \widehat{c}}\right\}=\left\{q_{1}^{(k)}, \ldots, q_{2 c_{k}}^{(k)}\right\}$. Note that $\left\{p_{1}, \ldots, p_{2 \widehat{c}}\right\} \subset\left\{a_{1}, \ldots, a_{k}\right\} \dot{\cup}\left\{d_{t+1-k}, \ldots, d_{t}\right\}$ and $\left\{q_{1}, \ldots, q_{2 \widehat{c}}\right\} \subset\left\{b_{1}, \ldots, b_{k}\right\} \cup \dot{\cup}\left\{c_{t+1-k}, \ldots, c_{t}\right\}$. Let $P_{1}$ (resp., $Q_{1}$ ) denote the topmost row of $P$ (resp., $Q$ ). Similarly let $P_{1}^{\prime}$ (resp., $Q_{1}^{\prime}$ ) denote the topmost row of $P^{\prime}$ (resp., $Q^{\prime}$ ).

Since $b$ is less than or equal to all elements of $\left\{b_{1}, \ldots, b_{k}\right\}$ and $b_{i}<c_{t+1-i} \forall i \in\{1, \ldots, t\}$, it follows that $b \leq$ all elements of $\left\{b_{1}, \ldots, b_{k}\right\} \dot{\cup}\left\{c_{t+1-k}, \ldots, c_{t}\right\}$. Therefore $a<b \leq q_{1}$, and hence by duality $c>d \geq p_{2 \widehat{c}}$. We consider two cases corresponding to the two ways in which $\left(P_{1}^{\prime}, Q_{1}^{\prime}\right)$ can be obtained from $\left(P_{1}, Q_{1}\right)$.

Case 1. $P_{1}^{\prime}$ is obtained by a bumping $p_{l}$ in $P_{1}$, for some $1 \leq$ $l \leq 2 \widehat{c}$; that is, $P_{1}^{\prime}=\left(P_{1} \backslash\left\{p_{l}\right\}\right) \dot{\cup}\{a\}$ and $Q_{1}^{\prime}=\left(Q_{1} \backslash\right.$ $\left.\left\{q_{2 \widehat{c}+1-l}\right\}\right) \dot{\cup}\{c\}$.

The fact that $a$ bumps $p_{l}$ implies that $a \leq p_{l}$ and $p_{l}<b$. Hence $a \leq p_{l}<b \leq q_{1}$, and therefore, by duality, we have $c \geq q_{2 \widehat{c}+1-l}>d \geq p_{2 \widehat{c}}$. This implies that $m(k+1)=m(k)$. For $j \in\{1, \ldots, m(k)\} \backslash\{l\}$, set $C_{k+1, j}^{(1)}=C_{k, j}^{(1)}$ and $C_{k+1, j}^{(2)}=C_{k, j}^{(2)}$ (Note that in these cases $p_{j}^{(k)}=p_{j}^{(k+1)}$.). We now consider the case when $j=l$. If $l=1$, then set $C_{k+1, l}^{(1)}=\{(a, b)\}$ and $C_{k+1, l}^{(2)}=\{(d, c)\}$. Otherwise consider the dual pair of chains $\left\{C_{k, l-1}^{(1)}, C_{k, l-1}^{(2)}\right\}$ in $\left\{U_{1}^{(k)}, U_{2}^{(k)}\right\}$. Let $C_{k+1, l}^{(1)}:=C_{k, l-1}^{(1)} \cup$ $\{(a, b)\}$ and $C_{k+1, l}^{(2)}:=C_{k, l-1}^{(2)} \cup\{(d, c)\}$. Note that the dual pair $\left\{C_{k+1, l}^{(1)}, C_{k+1, l}^{(2)}\right\}$ of chains in $\left\{U_{1}^{(k+1)}, U_{2}^{(k+1)}\right\}$ satisfies the required conditions.

Case 2. $P_{1}^{\prime}$ is obtained by adding $a$ to $P_{1}$ in position $l$ from the left and adding $d$ to $P_{1}$ at the rightmost end (after $p_{2 \widehat{c}}$ ); $Q_{1}^{\prime}$ is obtained from $Q_{1}$ by adding $b$ to the leftmost end of $Q_{1}$ and adding $c$ at the backward $l$ th position of $Q_{1}$. That is, $P_{1}^{\prime}=$ $P_{1} \dot{\cup}\{a, d\}=\left\{p_{1}<\cdots<p_{l-1}<a<p_{l}<\cdots<p_{2 \widehat{c}}<d\right\}$, and $Q_{1}^{\prime} \dot{\cup}\{b, c\}=\left\{b<q_{1}<\cdots<q_{2 \widehat{c}+1-l}<c<q_{2 \widehat{c}+1-(l-1)}<\cdots<\right.$ $\left.q_{2 \widehat{c}}\right\}$.

Since $p_{l-1}<a<b<q_{1}$, it follows that $m(k) \geq(l-1)$. Note that $a<b \leq p_{l}$ (since $b>p_{l}$ would require that $a$ bumps $p_{l}$ in the bounded insertion process). Thus $m(k+1)=$ l. For $j \in\{1, \ldots, l-1\}$, set $C_{k+1, j}^{(1)}=C_{k, j}^{(1)}$ and $C_{k+1, j}^{(2)}=C_{k, j}^{(2)}$. 
Consider the dual pair $\left\{C_{k, l-1}^{(1)}, C_{k, l-1}^{(2)}\right\}$ of chains in $\left\{U_{1}^{(k)}, U_{2}^{(k)}\right\}$. Let $C_{k+1, l}^{(1)}:=C_{k, l-1}^{(1)} \cup\{(a, b)\}$ and $C_{k+1, l}^{(2)}:=C_{k, l-1}^{(2)} \cup\{(d, c)\}$. Note that the dual pair $\left\{C_{k+1, l}^{(1)}, C_{k+1, l}^{(2)}\right\}$ of chains in $\left\{U_{1}^{(k+1)}, U_{2}^{(k+1)}\right\}$ satisfies the required conditions.

Lemma 42. Let $(P, Q)$ be a negative skew-symmetric notched bitableau. Let $a, b, c$, and $d$ be integers such that $(P, Q) \stackrel{b, c}{\leftarrow}$ $a, d$ is well defined. Let $\left(P^{\prime}, Q^{\prime}\right):=(P, Q) \stackrel{b, c}{\leftarrow} a, d$. Let $P_{1}$ (resp., $Q_{1}$ ) denote the topmost row of $P$ (resp., $\left.Q\right)$. Similarly let $P_{1}^{\prime}$ (resp., $Q_{1}^{\prime}$ ) denote the topmost row of $P^{\prime}$ (resp., $Q^{\prime}$ ). Let $\left\{p_{1}, \ldots, p_{2 \widehat{c}}\right\}$ (resp., $\left\{q_{1}, \ldots, q_{2 \widehat{c}}\right\}$ ) denote $P_{1}$ (resp., $Q_{1}$ ), both listed in increasing order. Suppose $P_{1}^{\prime}$ is obtained by a bumping $p_{l}$ in $P_{1}$, for some $1 \leq l \leq 2 \widehat{c}$; that is, suppose $P_{1}^{\prime}=\left(P_{1} \backslash\left\{p_{l}\right\}\right) \dot{\cup}\{a\}$ and $Q_{1}^{\prime}=\left(Q_{1} \backslash\left\{q_{2 \widehat{c}+1-l}\right\}\right) \dot{\cup}\{c\}$. Let $\left\{C_{1}, C_{2}\right\}$ be a dual pair of negative chains in $\mathbb{N}^{2}$ which satisfies condition (\#\#) with respect to the bitableau $\left(P^{\prime}, Q^{\prime}\right)$ and the integer l. Let $C_{1}=\left\{\left(e_{1}, f_{1}\right), \ldots,\left(e_{r}, f_{r}\right),(a, b)\right\}$ and $C_{2}=\left\{(d, c),\left(g_{r}, h_{r}\right), \ldots,\left(g_{1}, h_{1}\right)\right\}$, where $e_{1}<\cdots<e_{r}<$ $a<p_{l}<b<f_{r}<\cdots<f_{1}$ and $h_{1}>\cdots>h_{r}>c>$ $q_{2 \widehat{c}+1-l}>d>g_{r}>\cdots>g_{1}$. Then for $z$ such that $a \leq z<p_{l}$ or $q_{2 \widehat{c}+1-l} \leq z<c$, one has

$$
\left|\left(\left(P_{C_{1}, C_{2}}, Q_{C_{1}, C_{2}}\right)_{(1)}^{\text {up }}-\left(P_{C_{1}, C_{2}}, Q_{C_{1}, C_{2}}\right)_{(2)}^{\text {up }}\right)^{\leq z}\right| \geq\left|\left(P_{1}^{\prime}-Q_{1}^{\prime}\right)^{\leq z}\right| .
$$

Proof. Note that for $a \leq z<p_{l}$, we have $\mid\left(\left(P_{C_{1}, C_{2}}\right.\right.$, $\left.\left.Q_{C_{1}, C_{2}}\right)_{(1)}^{\text {up }}-\left(P_{C_{1}, C_{2}}, Q_{C_{1}, C_{2}}\right)_{(2)}^{\text {up }}\right)^{\leq z}|=|(\{$ topmost row of $\left.P_{C_{1}, C_{2}}\right\}-$ topmost row of $\left.\left.Q_{C_{1}, C_{2}}\right\}\right)^{\leq z}|=|(\{$ topmost row of $\left.P_{C_{1}, C_{2}}\right\} \dot{\cup}\left(\mathbb{N} \backslash\right.$ topmost row of $\left.\left.\left.Q_{C_{1}, C_{2}}\right\}\right)\right)^{\leq z} \mid=$ $\mid\left\{\text { topmost row of } P_{C_{1}, C_{2}}\right\}^{\leq z} \dot{U}\{\mathbb{N}\}^{\leq z} \mid \geq \chi_{l}+z \geq l+z$, where the last equality (not inequality!) is because $b \leq$ all entries in $Q_{C_{1}, C_{2}}$ and $a \leq z<p_{l}<b$ (All the other inequalities and equalities being obvious.).

Also, $p_{1}<\cdots<p_{l-1}<a<p_{l}<b \leq q_{1}<\cdots<q_{2 \widehat{c}}$, and $b<c$. Thus for $a \leq z<p_{l},\left|\left(P_{1}^{\prime}-Q_{1}^{\prime}\right)^{\leq z}\right|=\left|\left(P_{1}^{\prime} \dot{\cup}\left(\mathbb{N} \backslash Q_{1}^{\prime}\right)\right)^{\leq z}\right|=$ $\left|\left(P_{1}^{\prime} \dot{\cup} \mathbb{N}\right)^{\leq z}\right|=l+z$.

Hence we are done in the case $a \leq z<p_{l}$. Now for $z$ such that $q_{2 \widehat{c}+1-l} \leq z<c$, we need to show that

$$
\begin{aligned}
& \mid\left(\left\{\text { topmost row of } P_{C_{1}, C_{2}}\right\}\right. \\
& \left.\quad \dot{U}\left(\mathbb{N} \backslash\left\{\text { topmost row of } Q_{C_{1}, C_{2}}\right\}\right)\right)^{\leq z} \mid \\
& \quad \geq\left|\left(P_{1}^{\prime} \dot{\cup}\left(\mathbb{N} \backslash Q_{1}^{\prime}\right)\right)^{\leq z}\right| .
\end{aligned}
$$

Recall that $P_{1}^{\prime}=\left(P_{1} \backslash\left\{p_{l}\right\}\right) \dot{\cup}\{a\}$ and $Q_{1}^{\prime}=\left(Q_{1} \backslash\right.$ $\left.\left\{q_{2 \widehat{c}+1-l}\right\}\right) \dot{\cup}\{c\}$. Since $\left(P^{\prime}, Q^{\prime}\right)=(P, Q) \stackrel{b, c}{\leftarrow} a, d$, therefore $d \geq$ all entries of $P^{\prime}$. Hence $d \geq$ all entries of $P_{1}^{\prime}$. On the other hand, since $a<p_{l}<b$, it follows from duality that $c>q_{2 \widehat{c}+1-l}>d$. So we have $p_{1}<\cdots<p_{l-1}<a<p_{l+1}<\cdots<p_{2 \widehat{c}} \leq d<$ $q_{2 \widehat{c}+1-l}<c<q_{2 \widehat{c}+1-(l-1)}<\cdots<q_{2 \widehat{c}}$. It is now easy to observe that for $z$ such that $q_{2 \hat{c}+1-l} \leq z<c$, the number of elements in $Q_{1}^{\prime}$ which are $\leq z$ is $2 \widehat{c}-l$. Hence the number of elements in $\mathbb{N} \backslash Q_{1}^{\prime}$ which are $\leq z$ will be $z-(2 \widehat{c}-l)$.
It is also clear that all the elements of $P_{1}^{\prime}$ are $\leq z$, and there are $2 \widehat{c}$ many elements in $P_{1}^{\prime}$. Therefore,

$$
\begin{aligned}
\left|\left(P_{1}^{\prime} \dot{\cup}\left(\mathbb{N} \backslash Q_{1}^{\prime}\right)\right)^{\leq z}\right| & =2 \widehat{c}+(z-(2 \widehat{c}-l)) \\
& =2 \widehat{c}+z-2 \widehat{c}+l=z+l .
\end{aligned}
$$

Let $\alpha_{1}<\cdots<\alpha_{2 \widetilde{c}}$ and $\beta_{1}<\cdots<\beta_{2 \widetilde{c}}$ denote the topmost rows of $P_{C_{1}, C_{2}}$ and $Q_{C_{1}, C_{2}}$, respectively. It follows from the algorithm of OBRSK applied on $\left\{C_{1}, C_{2}\right\}$ that $d \geq \alpha_{2 \widehat{c}}>$ $\cdots>\alpha_{1}$. On the other hand, since $a<p_{l}<b$, it follows from duality that $c>q_{2 \widehat{c}+1-l}>d$. Hence combining all these, we have $c>q_{2 \widehat{\mathrm{c}}+1-l}>d \geq \alpha_{2 \widetilde{c}}>\alpha_{1}$. So for $z$ such that $q_{2 \widehat{c}+1-l} \leq z<c$, the number of elements in the topmost row of $P_{C_{1}, C_{2}}$ which are $\leq z$ is $2 \widetilde{c}$.

Since $\left\{C_{1}, C_{2}\right\}$ satisfies condition (\#\#) with respect to the bitableau $\left(P^{\prime}, Q^{\prime}\right)$ and the integer $l$, it follows that there exists an integer $\chi_{l}(\geq l)$ such that the $\chi_{l}$ th entry (counting from left to right) of the topmost row of $P_{C_{1}, C_{2}}$ is $a$. Hence it follows from duality that the backward $\chi_{l}$ th entry (i.e., the $\chi_{l}$ th entry counting from right to left) of the topmost row of $Q_{C_{1}, C_{2}}$ is $c$. Therefore for $z$ such that $q_{2 \widehat{c}+1-l} \leq z<c$, the number of elements in the topmost row of $Q_{C_{1}, C_{2}}$ which are $\leq z$ is equal to $X_{0}$, where $X_{0}$ is some nonnegative integer such that $X_{0} \leq$ $2 \widetilde{c}-\chi_{l}$. But $\chi_{l} \geq l$, hence $-\chi_{l} \leq-l$, and therefore $X_{0} \leq 2 \widetilde{c}-\chi_{l} \leq$ $2 \widetilde{c}-l$.

Therefore, the number of elements in $(\mathbb{N} \backslash$ topmost row of $\left.Q_{C_{1}, C_{2}}\right\}$ ) which are $\leq z$ is $z-X_{0}$. Hence $\mid\left(\left\{\right.\right.$ topmost row of $\left.P_{C_{1}, C_{2}}\right\} \dot{\cup}(\mathbb{N} \backslash$ topmost row of $\left.\left.\left.Q_{C_{1}, C_{2}}\right\}\right)\right)^{\leq z} \mid=2 \widetilde{c}+z-X_{0} \geq 2 \widetilde{c}+z-(2 \widetilde{c}-l)=z+l=$ $\left|\left(P_{1}^{\prime} \dot{\cup}\left(\mathbb{N} \backslash Q_{1}^{\prime}\right)\right)^{\leq z}\right|$.

\section{References}

[1] R. P. Stanley, "Some combinatorial aspects of the Schubert calculus," in Combinatoire et représentation du groupe symétrique, vol. 579 of Lecture Notes in Mathematics, Springer, Berlin, Germany, 1977.

[2] W. V. D. Hodge and D. Pedoe, Methods of Algebraic Geometry, vol. 1 of Cambridge Mathematical Library, Cambridge University Press, Cambridge, UK, 1994.

[3] K. N. Raghavan and S. Upadhyay, "Initial ideals of tangent cones to Schubert varieties in orthogonal Grassmannians," Journal of Combinatorial Theory Series A, vol. 116, no. 3, pp. 663-683, 2009.

[4] B. Sturmfels, "Gröbner bases and Stanley decompositions of determinantal rings," Mathematische Zeitschrift, vol. 205, no. 1, pp. 137-144, 1990.

[5] J. Herzog and N. V. Trung, "Gröbner bases and multiplicity of determinantal and Pfaffian ideals," Advances in Mathematics, vol. 96, no. 1, pp. 1-37, 1992.

[6] V. Kodiyalam and K. N. Raghavan, "Hilbert functions of points on Schubert varieties in Grassmannians," Journal of Algebra, vol. 270, no. 1, pp. 28-54, 2003.

[7] V. Kreiman and V. Lakshmibai, Monomial bases and applications for Richardson and Schubert varieties in ordinary and affine Grassmannians [Ph.D. thesis], Northeastern University, 2003.

[8] V. Kreiman, "Local properties of Richardson varieties in the Grassmannian via a bounded Robinson-Schensted-Knuth correspondence," Journal of Algebraic Combinatorics, vol. 27, no. 3, pp. 351-382, 2008. 
[9] K. N. Raghavan and S. Upadhyay, "Hilbert functions of points on Schubert varieties in orthogonal Grassmannians," Journal of Algebraic Combinatorics, vol. 31, no. 3, pp. 355-409, 2010.

[10] V. Lakshmibai and C. S. Seshadri, "Geometry of G/P-II. The work of de Concini and Procesi and the basic conjectures," Proceedings of the Indian Academy of Sciences A, vol. 87, no. 2, pp. 1-54, 1978. 


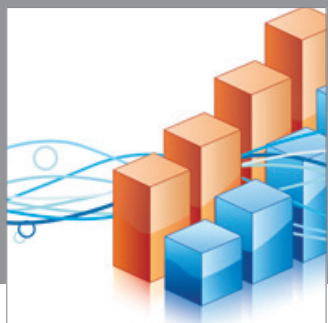

Advances in

Operations Research

mansans

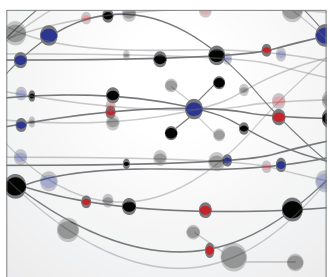

The Scientific World Journal
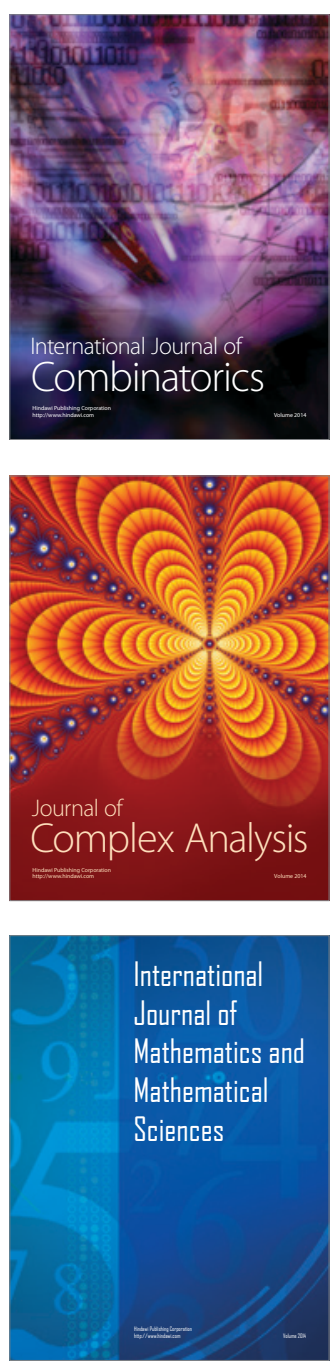
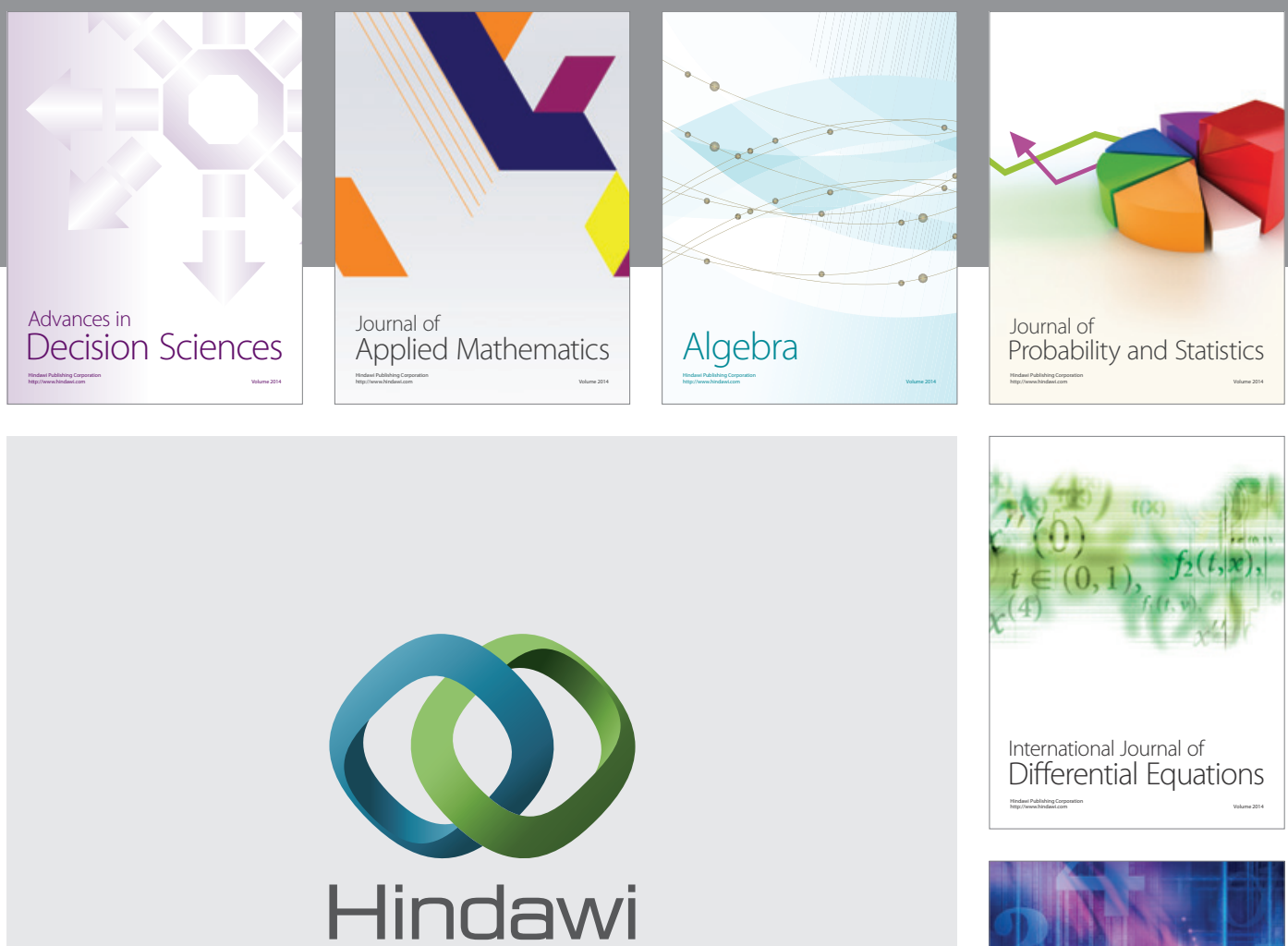

Submit your manuscripts at http://www.hindawi.com
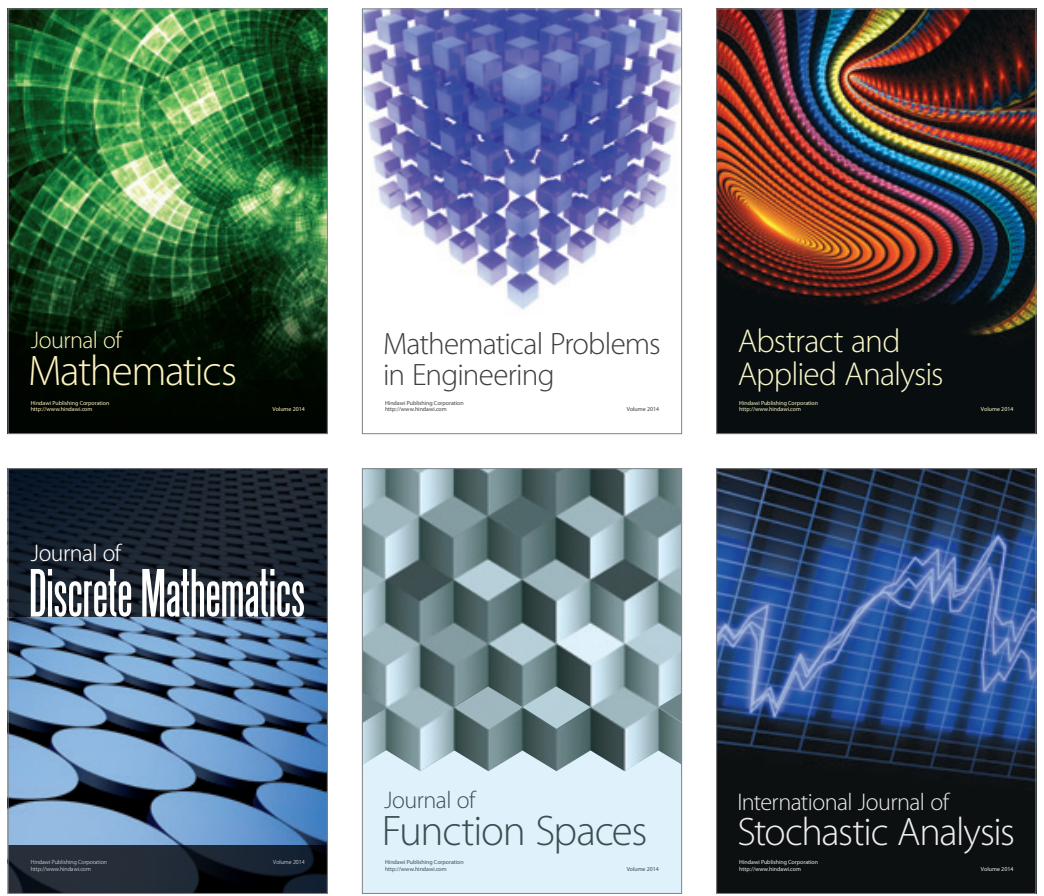

Journal of

Function Spaces

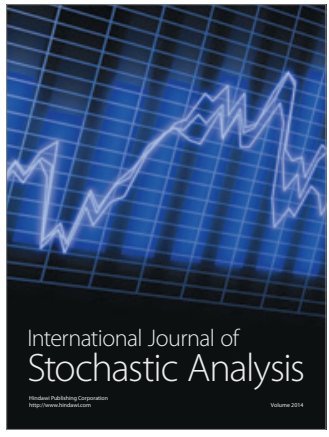

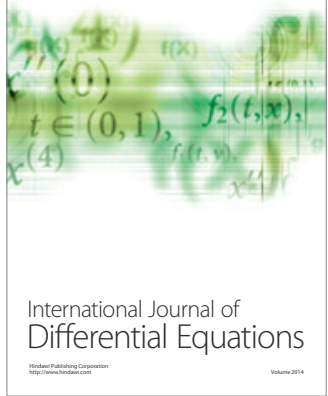
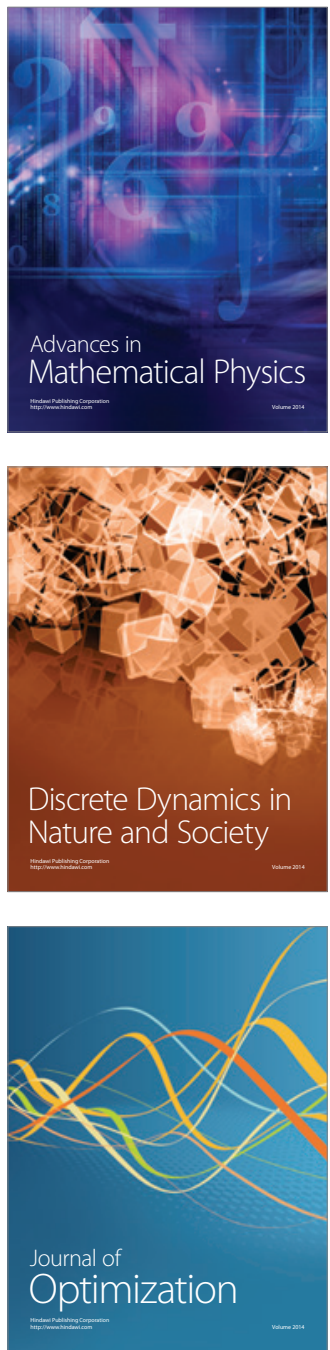